\title{
Barriers to Reallocation and Economic Growth: The Effects of Firing Costs
}

\author{
Toshihiko Mukoyama and Sophie Osotimehin
}

Online Appendix

\section{A Stationary distribution}

The stationary measure is the fixed point of the mapping $f \rightarrow \mathbf{T} f$, where $\mathbf{T} f$ gives the probability for the next period state given that the current state is drawn according to the probability measure $f$. The mass of firms in the set $\left[0, q^{\prime}\right] \times\left[0, \alpha^{\prime}\right] \times\left[0, \ell^{\prime}\right]$ next period is given by

$$
\int_{0}^{\alpha^{\prime}} \int_{0}^{\ell^{\prime}} \int_{0}^{\hat{q}^{\prime}} \mathbf{T} f(\hat{q}, \alpha, \ell) d \hat{q} d \alpha d \ell=(1-\delta)\left[(1-\mu) M_{s}\left(\hat{q}^{\prime}, \alpha^{\prime}, \ell^{\prime}\right)+M_{e}\left(\hat{q}^{\prime}, \alpha^{\prime}, \ell^{\prime}\right)\right] .
$$

The first term $M_{s}$ is the mass of non-displaced firms.

$$
\begin{aligned}
M_{s}\left(\hat{q}^{\prime}, \alpha^{\prime}, \ell^{\prime}\right)= & \int_{0}^{\alpha^{\prime}} \int_{\alpha} \int_{\hat{q} /\left(1+g_{q}\right) \leq \hat{q}^{\prime}} \int_{\mathcal{L}^{\prime}(\hat{q}, \alpha, \ell) \leq \ell^{\prime}} \zeta_{\alpha}\left(\alpha^{\prime}\right)\left(1-\mathcal{X}_{I}(\hat{q}, \alpha, \ell)\right) f(\hat{q}, \alpha, \ell) d \hat{q} d \ell d \alpha d \alpha^{\prime} \\
& +\int_{0}^{\alpha^{\prime}} \int_{\alpha} \int_{\left(1+\lambda_{I}\right) \hat{q} /\left(1+g_{q}\right)} \int_{\mathcal{L}^{\prime}(\hat{q}, \alpha, \ell) \leq \ell^{\prime}} \zeta_{\alpha}\left(\alpha^{\prime}\right) \mathcal{X}_{I}(\hat{q}, \alpha, \ell) f(\hat{q}, \alpha, \ell) d \hat{q} d \ell d \alpha d \alpha^{\prime}
\end{aligned}
$$

The second term $M_{e}$ is the mass of entering firms, which includes firms entering on inactive products and firms entering on existing products:

$$
\begin{aligned}
M_{e}\left(\hat{q}^{\prime}, \alpha^{\prime}, \ell^{\prime}\right)= & \mu(1-N) \int_{0}^{\alpha^{\prime}} \int_{\alpha} \int_{\left(1+\lambda_{E}\right) \hat{q} /\left(1+g_{q}\right) \leq \hat{q}^{\prime}} h(\hat{q}) \bar{\zeta}\left(\alpha^{\prime}\right) d \hat{q} d \alpha^{\prime} \\
& +\mu \int_{0}^{\alpha^{\prime}} \int_{\alpha} \int_{\left(1+\lambda_{E}\right) \hat{q} /\left(1+g_{q}\right) \leq \hat{q}^{\prime}} \int \zeta_{\alpha}\left(\alpha^{\prime}\right) f(\hat{q}, \alpha, \ell) d \hat{q} d \ell d \alpha d \alpha^{\prime}
\end{aligned}
$$

where $\zeta_{\alpha}\left(\alpha^{\prime}\right)$ is the distribution of the next period transitory shock conditional on the current period's value and $\bar{\zeta}\left(\alpha^{\prime}\right)$ is the invariant distribution of the transitory shock.

The expression of the stationary distribution is simpler when the model is rewritten in deviation to the frictionless values (see section II.B) and when the transitory shock $\alpha$ is i.i.d. as assumed in section III. In that case, the stationary distribution can then be rewritten as a function of the deviation of labor from its frictionless value $\tilde{\ell}$ instead of $\ell$, and the next period transitory shock draw becomes independent of next-period productivity and labor states. 
With these two modifications, $M_{s}$ becomes

$$
\begin{aligned}
M_{s}\left(\hat{q}^{\prime}, \alpha^{\prime}, \tilde{\ell}^{\prime}\right)=\Upsilon\left(\alpha^{\prime}\right) & {\left[\int_{\alpha} \int_{\hat{q} /\left(1+g_{q}\right) \leq \hat{q}^{\prime}} \int_{\mathcal{L}^{\prime}(\alpha, \ell) \leq \tilde{\ell}^{\prime}}\left(1-\mathcal{X}_{I}(\alpha, \tilde{\ell})\right) f(\hat{q}, \alpha, \tilde{\ell}) d \hat{q} d \alpha d \tilde{\ell}\right.} \\
& \left.+\int_{\alpha} \int_{\left(1+\lambda_{I}\right) \hat{q} /\left(1+g_{q}\right)} \int_{\mathcal{L}^{\prime}(\alpha, \tilde{\ell}) \leq \tilde{\ell}^{\prime}} \mathcal{X}_{I}(\alpha, \tilde{\ell}) f(\hat{q}, \alpha, \tilde{\ell}) d \hat{q} d \alpha d \tilde{\ell}\right]
\end{aligned}
$$

The mass of entrants $M_{e}$ can be rewritten as

$$
\begin{aligned}
M_{e}\left(\hat{q}^{\prime}, \alpha^{\prime}, \tilde{\ell}^{\prime}\right)=\Upsilon\left(\alpha^{\prime}\right) & {\left[\mu(1-N) \int_{\alpha} \int_{\left(1+\lambda_{E}\right) \hat{q} /\left(1+g_{q}\right) \leq \hat{q}^{\prime}} h(\hat{q}) d \hat{q}\right.} \\
& \left.+\mu \int_{\alpha} \int_{\left(1+\lambda_{E}\right) \hat{q} /\left(1+g_{q}\right) \leq \hat{q}^{\prime}} \iint f(\hat{q}, \alpha, \tilde{\ell}) d \hat{q} d \tilde{\ell} d \alpha\right],
\end{aligned}
$$

where $\Upsilon$ is the cumulative distribution of the transitory shock.

\section{B Analytical characterizations}

This section characterizes the model without the firing tax and boils it down to a system of nonlinear equations. The derivations also serve as proofs for the Propositions.

\section{B.1 Model solution}

Note first that for a given $\mu$, the number of actively produced product, $N$, is calculated by (10). Recall that $\mu$ is an endogenous variable and is determined by the entrants' innovation:

$$
\mu=m x_{E}^{*}
$$

As we have seen, $x_{E}^{*}$ is given by

$$
x_{E}^{*}=\left(\frac{\phi}{\theta_{E}(\gamma-1)}\right)^{\frac{1}{\gamma}},
$$

and thus $\mu$ (and also $N$ ) is a function of $m$. In particular, note that $N$ is an increasing function of $m$.

Because there are no firing taxes, the previous period employment, $\ell$, is no longer a state variable. The measure of individual states can be written as $f(\hat{q}, \alpha)$, and because $\hat{q}$ and $\alpha$ are independent, we can write $f(\hat{q}, \alpha)=\bar{f}(\hat{q}) \zeta(\alpha)$. In particular, note that $\int \hat{q} \bar{f}(\hat{q}) d \hat{q}=N$, because $\hat{q}$ is the value of $q_{t}$ normalized by its average. We also assume $\zeta(\alpha)$ is such that $\int \alpha \zeta(\alpha) d \alpha=1$.

Without firing costs, labor can be adjusted freely. Thus, the intermediate-good firm's decision for $\ell^{\prime}$ is static:

$$
\max _{\ell^{\prime}} \hat{\pi} \equiv\left([\alpha \hat{q}]^{\psi} \ell^{\prime-\psi} \hat{Y}^{\psi}-\hat{w}\right) \ell^{\prime}
$$


From the first-order condition,

$$
\ell^{\prime}=\left(\frac{1-\psi}{\hat{w}}\right)^{\frac{1}{\psi}} \alpha \hat{q} \hat{Y}
$$

holds. Because $y=\ell^{\prime}$, we can plug this into the definition of $\hat{Y}$ :

$$
\hat{Y}=\left(\iint[\alpha \hat{q}]^{\psi} y^{1-\psi} \bar{f}(\hat{q}) \zeta(\alpha) d \hat{q} d \alpha\right)^{\frac{1}{1-\psi}} .
$$

This yields

$$
\hat{Y}=\hat{Y}\left(\frac{1-\psi}{\hat{w}}\right)^{\frac{1}{\psi}} N^{\frac{1}{1-\psi}}
$$

and therefore

$$
\hat{w}=(1-\psi) N^{\frac{\psi}{1-\psi}} .
$$

Recall that $N$ is a function of the endogenous variable $m$. Thus, $\hat{w}$ is also a function of $m$.

Combining the equations (2) and (3), we get

$$
\ell^{\prime}=\alpha \hat{q} \hat{Y} N^{-\frac{1}{1-\psi}}
$$

Integrating this across all active firms yields

$$
L=N^{-\frac{\psi}{1-\psi}} \hat{Y}
$$

One way of looking at this equation is that $\hat{Y}$ can be pinned down once we know $L$ and $N$ (and thus $L$ and $m$ ). Plugging (3) and (4) into (1) yields

$$
\hat{\pi}=\psi \alpha \hat{q} \frac{\hat{Y}}{N}
$$

Now, let us characterize the innovation decision of an intermediate-good firm. Recall that the value functions are

$$
\hat{Z}(\hat{q}, \alpha)=(1-\delta) \hat{V}^{s}(\hat{q}, \alpha)
$$

where

$$
\hat{V}^{s}(\hat{q}, \alpha)=\max _{x_{I}} \psi \alpha \hat{q} \frac{\hat{Y}}{N}-\theta_{I} \hat{q} x_{I}^{\gamma}+\beta(1-\mu) \hat{S}\left(x_{I}, \hat{q} /\left(1+g_{q}\right)\right)
$$

and

$\hat{S}\left(x_{I}, \hat{q} /\left(1+g_{q}\right)\right)=\left(1-x_{I}\right) \int \hat{Z}\left(\hat{q} /\left(1+g_{q}\right), \alpha^{\prime}\right) \zeta\left(\alpha^{\prime}\right) d \alpha^{\prime}+x_{I} \int \hat{Z}\left(\left(1+\lambda_{I}\right) \hat{q} /\left(1+g_{q}\right), \alpha^{\prime}\right) \zeta\left(\alpha^{\prime}\right) d \alpha^{\prime}$.

We start from making a guess that $\hat{Z}(\hat{q}, \alpha)$ takes the form

$$
\hat{Z}(\hat{q}, \alpha)=\mathcal{A} \alpha \hat{q}+\mathcal{B} \hat{q}
$$


where $\mathcal{A}$ and $\mathcal{B}$ are constants. With this guess, the first-order condition in (5) for $x_{I}$ is

$$
\gamma \theta_{I} \hat{q} x_{I}^{\gamma-1}=\frac{\beta(1-\mu) \lambda_{I}(\mathcal{A}+\mathcal{B}) \hat{q}}{1+g_{q}} .
$$

Thus,

$$
x_{I}=\left(\frac{\beta(1-\mu) \lambda_{I}(\mathcal{A}+\mathcal{B})}{\left(1+g_{q}\right) \gamma \theta_{I}}\right)^{\frac{1}{\gamma-1}},
$$

and $x_{I}$ is constant across $\hat{q}$ and $\alpha$. Substituting for $x_{I}$, the value function can be written as

$$
\hat{Z}(\hat{q}, \alpha)=(1-\delta)\left(\psi \alpha \hat{q} \frac{\hat{Y}}{N}-\theta_{I} \hat{q} x_{I}^{\gamma}+\beta(1-\mu) \frac{1+x_{I} \lambda_{I}}{1+g_{q}}(\mathcal{A}+\mathcal{B}) \hat{q}\right) .
$$

Thus, the guess is verified with

$$
\mathcal{A}=(1-\delta) \psi \frac{\hat{Y}}{N}
$$

and $\mathcal{B}$ solves

$\mathcal{B}=(1-\delta)\left(-\theta_{I} x_{I}^{\gamma}+\beta(1-\mu) \frac{1+x_{I} \lambda_{I}}{1+g_{q}}(\mathcal{A}+\mathcal{B})\right)=(1-\delta) \beta(1-\mu)\left(1+\frac{\gamma-1}{\gamma} \lambda_{I} x_{I}\right) \frac{\mathcal{A}+\mathcal{B}}{1+g_{q}}$,

where $x_{I}$ is given by (6). Therefore, we found that $x_{I}$ (and the coefficients of the function $\hat{Z}(\hat{q}, \alpha))$ is a function of the endogenous aggregate variables $\mu, g_{q}, \hat{Y}$, and $N$. We have already seen we can pin down $\mu$ and $N$ if we know $m$, and $\hat{Y}$ can be pinned down if we know $m$ and $L$.

We now turn to the growth rate of productivity $g_{q}$. As we have seen above, the transitory shock $\alpha$ does not affect the innovation decision and can therefore be ignored when calculating the transition function of $q_{t}$. Consider the measure of productivity (without the normalization) $q_{t}$ for active products, $z\left(q_{t}\right)$. A fraction $(1-\mu) x_{I}(1-\delta)$ of active lines are products that have been innovated upon by incumbents and the fraction $\left(1-\mu-(1-\mu) x_{I}\right)(1-\delta)$ is owned by the incumbents but the innovation was unsuccessful. The fraction $\mu(1-\delta)$ of active products is innovated upon by entrants. The fraction $\mu(1-\delta)$ of inactive products is innovated upon by entrants. The productivity distribution of inactive product lines is $h\left(q_{t} / \bar{q}_{t}\right)$ rather than $\bar{f}\left(q_{t} / \bar{q}_{t}\right) / N$. Thus, $g_{q}$ can be calculated from

$$
1+g_{q}=(1-\delta)\left[\left(1+\lambda_{I} x_{I}\right)(1-\mu)+\left(1+\lambda_{E}\right) \mu+\left(1+\lambda_{E}\right) \mu \frac{1-N}{N} \frac{\bar{q}^{h}}{\bar{q}^{f}}\right],
$$

where $\bar{q}^{h}$ and $\bar{q}^{f}$ are averages of $q_{t}$ with respect to the distributions $h$ and $\bar{f}$. Thus, $\bar{q}^{h} / \bar{q}^{f}=\int q_{t} h\left(q_{t} / \bar{q}_{t}\right) d q_{t} / \int q_{t}\left[\bar{f}\left(q_{t} / \bar{q}_{t}\right) / N\right] d q_{t}=\int \hat{q} h(\hat{q}) d \hat{q} / \int \hat{q}[\bar{f}(\hat{q}) / N] d \hat{q}$. The first term is the productivity increase of the surviving incumbents, the second term is the entry into active products, and the last is the entry into inactive products. Using the expression for $N$ in (10) and the fact that $\bar{q}^{f}=1$,

$$
g_{q}=(1-\delta)\left[\left(1+\lambda_{I} x_{I}\right)(1-\mu)+\left(1+\lambda_{E}\right) \mu\right]+\delta\left(1+\lambda_{E}\right) \bar{q}^{h}-1 .
$$


Thus, $g_{q}$ can be written as a function of $\mu$ and $x_{I}$, and therefore $m$ and $L$.

Hence, we can determine all endogenous variables in the economy once we pin down $m$ and $L$. The values of $m$ and $L$ can be pinned down by two additional conditions: the labor-market equilibrium condition and the free-entry condition. To see this, let us first be explicit about each variable's (and each coefficient's) dependence on $m$ and $L$ : $\hat{w}(m), N(m), \hat{Y}(m, L), x_{I}(m, L), g_{q}(m, L), \mathcal{A}(m, L)$, and $\mathcal{B}(m, L)$. Also note that the total $\mathrm{R} \& \mathrm{D}, \hat{R}$, can be written as

$$
\hat{R}=\int \theta_{I} \hat{q} x_{I}(m, L)^{\gamma} \bar{f}(\hat{q}) d \hat{q}+m\left(\phi+\theta_{E} x_{E}^{\gamma}\right)=\theta_{I} N(m) x_{I}(m, L)^{\gamma}+m\left(\phi+\theta_{E} x_{E}^{\gamma}\right)
$$

and therefore we can write $\hat{R}(m, L)$.

The labor-market equilibrium condition is

$$
\frac{\hat{w}(m)}{\hat{Y}(m, L)-\hat{R}(m, L)}=\xi
$$

and the free-entry condition is

$$
\frac{\gamma \theta_{E} x_{E}^{\gamma-1}}{\beta}=\hat{\bar{V}}_{E}
$$

where

$$
\begin{aligned}
\hat{\bar{V}}_{E} & =\int\left[\int \hat{Z}\left(\left(1+\lambda_{E}\right) \hat{q} /\left(1+g_{q}\right), \alpha\right)(\bar{f}(\hat{q})+(1-N) h(\hat{q})) d \hat{q}\right] \zeta(\alpha) d \alpha \\
& =\int \frac{\mathcal{A}(m, L)+\mathcal{B}(m, L)}{1+g_{q}(m, L)}\left(1+\lambda_{E}\right) \hat{q}(\bar{f}(\hat{q})+(1-N) h(\hat{q})) d \hat{q} \\
& =\frac{\mathcal{A}(m, L)+\mathcal{B}(m, L)}{1+g_{q}(m, L)}\left(1+\lambda_{E}\right)\left[N(m)+(1-N(m)) \bar{q}^{h}\right] .
\end{aligned}
$$

\section{B.2 Productivity distribution}

The invariant distribution $\bar{f}(\hat{q})$ can be easily computed. The next-period mass at relative quality $\hat{q}$ is the sum of four components: (i) the incumbents' innovation, $(1-\delta)(1-$ $\mu) x_{I} \bar{f}\left(\left(1+g_{q}\right) \hat{q} /\left(1+\lambda_{I}\right)\right) d \hat{q}$; (ii) the entrants' innovation, $(1-\delta) \mu \bar{f}\left(\left(1+g_{q}\right) \hat{q} /\left(1+\lambda_{E}\right)\right) d \hat{q}$; (iii) the downgrade from products that were not innovated upon, $((1-\delta)(1-\mu-(1-$ $\left.\mu) x_{I}\right) \bar{f}\left(\left(1+g_{q}\right) \hat{q}\right) d \hat{q}$; and (iv) the entry from inactive products, $(1-\delta) \mu(1-N) h(\hat{q} /(1+$ $\left.\left.\lambda_{E}\right)\right) d q$. The sum of these four components must be equal to $\bar{f}(\hat{q}) d \hat{q}$ along the stationary growth path.

We can characterize the right tail of the distribution analytically, when the distribution $h(\hat{q})$ is bounded. Let the density function of the stationary distribution be $s(\hat{q}) \equiv \bar{f}(\hat{q}) / N$. Because $h(\hat{q})$ is bounded, there is no direct inflow from the inactive product lines at the right tail.

Consider the point $\hat{q}$ and the interval $\Delta$ around that point. The outflow from that interval is $s(\hat{q}) \Delta$ because all the firms will either move up, move down, or exit.

The inflow comes from two sources. The first source is the mass of firms that innovated. Innovation is either done by incumbents or entrants. Let $\gamma_{i} \equiv\left(1+\lambda_{I}\right) /\left(1+g_{q}\right)>1$ 
be the (adjusted) improvement of $\hat{q}$ after innovation by an incumbent. The probability of innovation by an incumbent is $(1-\delta)(1-\mu) x_{I}$ and the corresponding mass of this inflow is $(1-\delta)(1-\mu) x_{I} s\left(\hat{q} / \gamma_{i}\right) \Delta / \gamma_{i}$. Similarly, letting $\gamma_{e} \equiv\left(1+\lambda_{E}\right) /\left(1+g_{q}\right)>1$ be the improvement of $\hat{q}$ after innovation by an entrant, the mass of the inflow due to the entrants' innovation is $(1-\delta) \mu s\left(\hat{q} / \gamma_{e}\right) \Delta / \gamma_{e}$. The second source of inflow is the surviving firms that did not innovate. With probability $(1-\delta)(1-\mu)\left(1-x_{I}\right)$, incumbents firms are not successful at innovating. Let $\gamma_{n} \equiv 1 /\left(1+g_{q}\right)<1$ be the (adjusted) quality ratio when there is no innovation. The corresponding mass of this inflow is $(1-\delta)(1-\mu)\left(1-x_{I}\right) s\left(\hat{q} / \gamma_{n}\right) \Delta / \gamma_{n}$.

In the stationary distribution, the inflows are equal to the outflows, and therefore

$$
s(\hat{q}) \Delta=(1-\delta)\left[(1-\mu) x_{I} s\left(\frac{\hat{q}}{\gamma_{i}}\right) \frac{\Delta}{\gamma_{i}}+\mu s\left(\frac{\hat{q}}{\gamma_{e}}\right) \frac{\Delta}{\gamma_{e}}+\left(1-\mu-(1-\mu) x_{I}\right) s\left(\frac{\hat{q}}{\gamma_{n}}\right) \frac{\Delta}{\gamma_{n}}\right],
$$

or

$$
s(\hat{q})=(1-\delta)\left[(1-\mu) x_{I} s\left(\frac{\hat{q}}{\gamma_{i}}\right) \frac{1}{\gamma_{i}}+\mu s\left(\frac{\hat{q}}{\gamma_{e}}\right) \frac{1}{\gamma_{e}}+\left(1-\mu-(1-\mu) x_{I}\right) s\left(\frac{\hat{q}}{\gamma_{n}}\right) \frac{1}{\gamma_{n}}\right],
$$

Guess that the right-tail of the density function is Pareto and has the form $s(x)=$ $F x^{-(\kappa+1)}$. The parameter $\kappa>0$ is the shape parameter, and the expected value of $x$ exists only if $\kappa>1$. Plugging this guess into the expression above yields

$$
\begin{aligned}
& F \hat{q}^{-(\kappa+1)}= \\
& \qquad \begin{array}{r}
(1-\delta)\left[(1-\mu) x_{I} F\left(\frac{\hat{q}}{\gamma_{i}}\right)^{-(\kappa+1)} \frac{1}{\gamma_{i}}+\mu F\left(\frac{\hat{q}}{\gamma_{e}}\right)^{-(\kappa+1)} \frac{1}{\gamma_{e}}\right. \\
\left.\quad+\left(1-\mu-(1-\mu) x_{I}\right) F\left(\frac{\hat{q}}{\gamma_{n}}\right)^{-(\kappa+1)} \frac{1}{\gamma_{n}}\right],
\end{array}
\end{aligned}
$$

or

$$
1=(1-\delta)\left[(1-\mu) x_{I} \gamma_{i}^{\kappa}+\mu \gamma_{e}^{\kappa}+\left(1-\mu-(1-\mu) x_{I}\right) \gamma_{n}^{\kappa}\right]
$$

The parameter $\kappa$ is the solution of this equation.

\section{B.3 Growth rate}

The growth rate of aggregate productivity is given by

$$
g_{q}=(1-\delta)\left[\left(1+\lambda_{I} x_{I}\right)(1-\mu)+\left(1+\lambda_{E}\right) \mu\right]+\delta\left(1+\lambda_{E}\right) \bar{q}^{h}-1
$$

where $\bar{q}^{h}$ is the average relative productivity of inactive product lines. This can be shown by a simple accounting relation. Let the measure of $q_{t}$ (without normalization) for active products be $\bar{f}\left(q_{t} / \bar{q}_{t}\right)$. Innovation by incumbents occurs on a fraction $(1-\mu) x_{I}(1-\delta)$ of active product lines; no innovation occurs on a fraction $\left(1-\mu-(1-\mu) x_{I}\right)(1-\delta)$ of active lines. Entrants innovate on a fraction $\mu(1-\delta)$ of active products. Among the inactive products, the fraction $\mu(1-\delta)$ becomes active from the innovation by entrants, 
but it is an upgrade from the distribution $h\left(q_{t} / \bar{q}_{t}\right)$ rather than $\bar{f}\left(q_{t} / \bar{q}_{t}\right) / N$. Thus, $g_{q}$ can be calculated from

$$
1+g_{q}=(1-\delta)\left[\left(1+\lambda_{I} x_{I}\right)(1-\mu)+\left(1+\lambda_{E}\right) \mu+\left(1+\lambda_{E}\right) \mu \frac{1-N}{N} \frac{\bar{q}^{h}}{\bar{q}^{f}}\right] .
$$

Here, $\bar{q}^{h}$ and $\bar{q}^{f}$ are averages of $q_{t}$ with respect to the distributions $h$ and $f$. Thus, $\bar{q}^{h} / \bar{q}^{f}=$ $\int q_{t} h\left(q_{t} / \bar{q}_{t}\right) d q_{t} / \int q_{t}\left[\bar{f}\left(q_{t} / \bar{q}_{t}\right) / N\right] d q_{t}=\int \hat{q} h(\hat{q}) d \hat{q} / \int \hat{q}[\bar{f}(\hat{q}) / N] d \hat{q}$. Combining this with the expression for $N$ in (10) and the fact that $\bar{q}^{f}=1$ yields the above result.

\section{B.4 Details of section II.B}

Under the notations of section II.B, the period profit (9) can be rewritten as

$$
\begin{aligned}
& \hat{\Pi}\left(\hat{q}, \alpha, \ell, \ell^{\prime}, x_{I}\right)= \\
& \quad\left(\left[\frac{\alpha}{\Omega(\hat{w}, \hat{Y})}\right]^{\psi} \tilde{\ell}^{\prime-\psi} \hat{Y}^{\psi}-\hat{w}\right) \hat{q} \Omega(\hat{w}, \hat{Y}) \tilde{\ell}^{\prime}-\theta_{I} \hat{q} x_{I}^{\gamma}-\tau \hat{w} \max \left\langle 0, \hat{q} \Omega(\hat{w}, \hat{Y}) \tilde{\ell}-\hat{q} \Omega(\hat{w}, \hat{Y}) \tilde{\ell^{\prime}}\right\rangle .
\end{aligned}
$$

Thus, this is linear in $\hat{q}$, and can be rewritten as $\hat{q} \tilde{\Pi}\left(\alpha, \tilde{\ell}, \tilde{\ell}^{\prime}, x_{I}\right)$, where

$$
\tilde{\Pi}\left(\alpha, \tilde{\ell}, \tilde{\ell}^{\prime}, x_{I}\right) \equiv\left(\left[\frac{\alpha}{\Omega(\hat{w}, \hat{Y})}\right]^{\psi} \tilde{\ell}^{\prime-\psi} \hat{Y}^{\psi}-\hat{w}\right) \Omega(\hat{w}, \hat{Y}) \tilde{\ell}^{\prime}-\theta_{I} x_{I}^{\gamma}-\tau \Omega(\hat{w}, \hat{Y}) \hat{w} \max \left\langle 0, \tilde{\ell}-\tilde{\ell}^{\prime}\right\rangle .
$$

Because the period return function is linear in $\hat{q}$, it is straightforward to show that

all value functions are linear in $\hat{q}$. Defining $\tilde{Z}(\alpha, \tilde{\ell})$ from $\hat{Z}(\hat{q}, \alpha, \ell)=\hat{q} \tilde{Z}(\alpha, \tilde{\ell})$, (7) can be rewritten as

$$
\tilde{Z}(\alpha, \tilde{\ell})=(1-\delta) \tilde{V}^{s}(\alpha, \tilde{\ell})+\delta \tilde{V}^{o}(\tilde{\ell}),
$$

where $\tilde{V}^{o}(\tilde{\ell})$ is from $\hat{V}^{o}(\ell)=\hat{q} \tilde{V}^{o}(\tilde{\ell})$ and thus

$$
\tilde{V}^{o}(\tilde{\ell})=-\tau \hat{w} \Omega(\hat{w}, \hat{Y}) \tilde{\ell}
$$

and $\tilde{V}^{s}(\alpha, \tilde{\ell})$ is from $\hat{V}^{s}(\hat{q}, \alpha, \ell)=\hat{q} \tilde{V}^{s}(\alpha, \tilde{\ell})$ with

$$
\tilde{V}^{s}(\alpha, \tilde{\ell})=\max _{\tilde{\ell}^{\prime} \geq 0, x_{I}}\left\{\tilde{\Pi}\left(\alpha, \tilde{\ell}, \tilde{\ell}^{\prime}, x_{I}\right)+\beta\left((1-\mu) \frac{\tilde{S}\left(x_{I}, \tilde{\ell}^{\prime}\right)}{1+g_{q}}-\mu \tau \hat{w} \Omega(\hat{w}, \hat{Y}) \tilde{\ell}^{\prime}\right)\right\} .
$$

Here, the expression $\tilde{S}\left(x_{I}, \tilde{\ell^{\prime}}\right) /\left(1+g_{q}\right)$ comes from $\hat{S}\left(x_{I}, \hat{q} /\left(1+g_{q}\right), \ell^{\prime}\right)=\hat{q} \tilde{S}\left(x_{I}, \tilde{\ell}^{\prime}\right) /\left(1+g_{q}\right)$. The linearity of the value functions implies

$$
\frac{\tilde{S}\left(x_{I}, \tilde{\ell}^{\prime}\right)}{1+g_{q}}=\left(1-x_{I}\right) E_{\alpha^{\prime}}\left[\tilde{Z}\left(\alpha^{\prime},\left(1+g_{q}\right) \tilde{\ell}^{\prime}\right)\right] \frac{1}{1+g_{q}}+x_{I} E_{\alpha^{\prime}}\left[\tilde{Z}\left(\alpha^{\prime}, \frac{\left(1+g_{q}\right) \tilde{\ell}^{\prime}}{1+\lambda_{I}}\right)\right] \frac{1+\lambda_{I}}{1+g_{q}}
$$

also holds. Here we used that

$$
\hat{Z}\left(\hat{q}^{\prime}, \alpha^{\prime}, \ell^{\prime}\right)=\hat{q}^{\prime} \tilde{Z}\left(\alpha^{\prime}, \frac{\ell^{\prime}}{\ell^{*}\left(\hat{q}^{\prime} ; \hat{w}^{\prime}, \hat{Y}^{\prime}\right)}\right)=\hat{q}^{\prime} \tilde{Z}\left(\alpha^{\prime}, \frac{\ell^{*}(\hat{q} ; \hat{w}, \hat{Y})}{\ell^{*}\left(\hat{q}^{\prime} ; \hat{w}^{\prime}, \hat{Y}^{\prime}\right)} \frac{\ell^{\prime}}{\ell^{*}(\hat{q} ; \hat{w}, \hat{Y})}\right)
$$


with $\hat{w}^{\prime}=\hat{w}, \hat{Y}^{\prime}=\hat{Y}$, and that $\ell^{*}(\hat{q} ; \hat{w}, \hat{Y}) / \ell^{*}\left(\hat{q}^{\prime} ; \hat{w}^{\prime}, \hat{Y}^{\prime}\right)=\hat{q} / \hat{q}^{\prime}$ yields

$$
\hat{Z}\left(\hat{q}^{\prime}, \alpha^{\prime}, \ell^{\prime}\right)=\hat{q}^{\prime} \tilde{Z}\left(\alpha^{\prime}, \frac{\hat{q}}{\hat{q}^{\prime}} \tilde{\ell}^{\prime}\right)
$$

for $\hat{q}^{\prime}=\hat{q} /\left(1+g_{q}\right)$ and $\hat{q}^{\prime}=\left(1+\lambda_{I}\right) \hat{q} /\left(1+g_{q}\right)$.

\section{Measuring the welfare loss of the growth decline}

To evaluate the size of the growth effect of the firing tax and to better compare our results with the literature, we conduct a back-of-the-envelope calculation of the consumptionequivalent welfare change in the entrant-driven case of the main text. We compare the balanced-growth welfare of consumers in the economies with and without the firing tax. ${ }^{1}$

The consumer's utility under balanced growth is

$$
\sum_{t=0}^{\infty} \beta^{t}\left[\log \left(\hat{C}(1+g)^{t}\right)-\xi L\right]
$$

which can be separated into two components:

$$
\sum_{t=0}^{\infty} \beta^{t}\left[\log \left(\hat{C}(1+g)^{t}\right)-\xi L\right]=\sum_{t=0}^{\infty} \beta^{t}\left[\log \left(\hat{C}(1+g)^{t}\right)\right]-\sum_{t=0}^{\infty} \beta^{t} \xi L .
$$

The first component is the effect of consumption, and the second component is the effect of labor. In our outcome with the entrant-driven case, $g, \hat{C}$, and $L$ all decline with the firing tax. Note the decline in $L$ leads to a welfare gain. We focus here on the welfare losses of the firing tax, and therefore abstract from the effects of the firing tax on labor. ${ }^{2}$

The consumption component of welfare can itself be separated in two parts

$$
\sum_{t=0}^{\infty} \beta^{t}\left[\log \left(\hat{C}(1+g)^{t}\right)\right]=\sum_{t=0}^{\infty} \beta^{t} \log (\hat{C})+\sum_{t=0}^{\infty} \beta^{t} \log \left((1+g)^{t}\right)
$$

We call the first term the level effect and the second term the growth effect on consumer welfare.

Let the consumption level and the growth rate be $\hat{C}_{0}$ and $g_{0}$ in the economy without the firing tax, and $\hat{C}_{1}$ and $g_{1}$ in the economy with the firing tax. We compute the welfare loss from the level effect and the growth effect of the firing tax as the permanent drop in consumption that would make the representative consumer in the economy without the firing tax indifferent between the two economies.

For the level effect, the permanent drop in consumption $\varphi_{L}$ is such that

$$
\sum_{t=0}^{\infty} \beta^{t} \log \left(\left(1-\varphi_{L}\right) \hat{C}_{0}\right)=\sum_{t=0}^{\infty} \beta^{t} \log \left(\hat{C}_{1}\right)
$$

\footnotetext{
${ }^{1}$ This is not a complete analysis of consumer welfare, because the comparison below does not take the transition dynamics into account. Our analysis here is meant to be illustrative.

${ }^{2}$ This approach (ignoring the change in disutility of labor and focusing on consumption) is similar to the approach that Lucas (1987) used in his welfare-cost calculation for the business cycles.
} 
Thus,

$$
\varphi_{L}=1-\exp \left(\log \left(\hat{C}_{1}\right)-\log \left(\hat{C}_{0}\right)\right)=1-\frac{\hat{C}_{1}}{\hat{C}_{0}} .
$$

For the growth effect, the permanent drop in consumption $\varphi_{G}$ is computed as

$$
\sum_{t=0}^{\infty} \beta^{t} \log \left(\left(1-\varphi_{G}\right)\left(1+g_{0}\right)^{t}\right)=\sum_{t=0}^{\infty} \beta^{t} \log \left(\left(1+g_{1}\right)^{t}\right) \text {. }
$$

This equation can be solved to obtain

$$
\varphi_{G}=1-\left(\frac{1+g_{1}}{1+g_{0}}\right)^{\frac{\beta}{1-\beta}} .
$$

In our experiment with entrant-driven case $(\tau=0.3)$, we find that $\varphi_{L}=1-\hat{w}_{1} / \hat{w}_{0}=$

$0.9 \%$ and $\varphi_{G}=1-\left(\frac{1.0139}{1.0148}\right)^{\frac{0.947}{1-0.947}}=1.6 \%$. The growth effect is larger than the level effect.

\section{Details of computation}

The computation solution consists of first guessing the values of the relevant aggregate variables, solving for the value function and the stationary distribution of firms, and then updating the guess. The procedure is as follows.

1. Construct a grid for productivity $\hat{q}$ and labor $\tilde{\ell}$. We use a $\log$ grid for $\hat{q}$ with 100 points between 0 and $10^{9}$. For $\tilde{\ell}$, we use a linear grid with 30 points between 0 and 4 .

2. Compute the innovation from entrants and the value from entry consistent with the free entry condition

$$
\begin{gathered}
x_{E}^{*}=\left(\frac{\phi}{\theta_{E}(\gamma-1)}\right)^{\frac{1}{\gamma}}, \\
\hat{\bar{V}}_{E}=\frac{\gamma \theta_{E}}{\beta} x_{E}^{\gamma-1} .
\end{gathered}
$$

3. Guess $\hat{Y}, \hat{w}, m$, and $g$. Given $m$, we can calculate the value of $\mu$ by $\mu=X_{E}=m x_{E}^{*}$.

4. Solve for the value function by iterating on the value function and using linear interpolation between grid points.

5. Using the optimal decision rules, solve for the stationary distribution $f(\hat{q}, \alpha, \tilde{\ell})$ by iterating over the density.

6. Then check if the equilibrium conditions are verified. The four conditions are the following 
(a) Aggregate output

$$
\hat{Y}=\left(\iint \alpha^{\psi}\left[\Omega(\hat{w}, \hat{Y}) \mathcal{L}^{\prime}(\alpha, \tilde{\ell})\right]^{1-\psi} \int \hat{q} f(\hat{q}, \alpha, \tilde{\ell}) d \hat{q} d \alpha d \tilde{\ell}\right)^{\frac{1}{1-\psi}}
$$

(b) Resource constraint

$$
\hat{Y}=\hat{C}+\hat{R}
$$

with $\hat{C}=\hat{w} / \xi$ and $\hat{R}=\theta_{I} \iint \mathcal{X}_{I}(\alpha, \tilde{\ell})^{\gamma} \int \hat{q} f(\hat{q}, \alpha, \tilde{\ell}) d \hat{q} d \alpha d \tilde{\ell}+m\left(\phi+\theta_{E} x_{E}^{\gamma}\right)$

(c) Consistency condition for productivity

$$
\frac{1}{N} \iiint \hat{q} f(\hat{q}, \alpha, \tilde{\ell}) d \alpha d \ell d q=1
$$

(d) Free-entry condition

$$
\hat{\bar{V}}_{E}=\frac{\gamma \theta_{E}}{\beta} x_{E}^{\gamma-1}
$$

where $^{3}$

$$
\hat{\bar{V}}_{E}=\int \tilde{Z}(\alpha, 0) \zeta(\alpha) d \alpha\left[N+(1-N) \int h(\hat{q}) d \hat{q}\right]\left(1+\lambda_{E}\right) /\left(1+g_{q}\right) .
$$

We use condition (a) to update the value for $\hat{w}$. When $\hat{w}$ is too high, aggregate output implied by the firms decision is too low. We use condition (b) to update the value for $\hat{Y}$. If $\hat{Y}$ is too high, the resource constraint is not satisfied. We update $g_{q}$ using condition (c). Intuitively, when $g_{q}$ is too small, the stationary density $f(\hat{q}, \alpha, \tilde{\ell})$ implies the values of $\hat{q}$ that are too large. To update the value of $m$, we use condition $(\mathrm{d})$. Because a large value of $m$ implies a large value of $\mu$, which a larger value of $m$ implies a lower value of $\tilde{Z}$. Thus, the value of $m$ affects the computed value of $\hat{\bar{V}}_{E}$, through $\tilde{Z}$.

7. Go back to Step 3, until convergence.

\section{E Details of the extensions and robustness checks of section IV}

\section{E.1 Extension 1: Persistent exogenous shocks}

This section complements section IV.A by giving more details on the calibration of the extension with persistent transitory shocks.

\footnotetext{
${ }^{3}$ Computed from

$$
\begin{aligned}
\hat{\bar{V}}_{E} & =\int\left[\int \hat{Z}\left(\left(1+\lambda_{E}\right) \hat{q} /\left(1+g_{q}\right), \alpha, 0\right)(\bar{f}(\hat{q})+(1-N) h(\hat{q})) d \hat{q}\right] \zeta(\alpha) d \alpha \\
& =\int\left[\int \hat{q} \tilde{Z}(\alpha, 0)\left(1+\lambda_{E}\right) /\left(1+g_{q}\right)(\bar{f}(\hat{q})+(1-N) h(\hat{q})) d \hat{q}\right] \zeta(\alpha) d \alpha .
\end{aligned}
$$
}




\section{E.1.1 Calibration}

We use the variance and autocovariance of establishment-level employment growth to identify the size of the shock $\varepsilon$ and the persistence parameter $\rho$. To give the intuition behind this strategy, let us assume that instead of following a discrete-valued Markov process, the exogenous productivity $\alpha$ follows an $\mathrm{AR}(1)$ process (in logs), that is, $\ln \alpha_{t}=$ $\varphi \ln \alpha_{t-1}+u_{t}$, where $u_{t}$ is i.i.d. with mean zero and variance $\sigma_{u}^{2}$. This assumption simplifies the expression of the variance and covariance of log employment changes. In the absence of firing costs, the employment of the firm is given by $\ell=\left(\frac{1-\psi}{\hat{w}}\right)^{\frac{1}{\psi}} \alpha \hat{q} \hat{Y}$, the variance of $\log$ employment changes is then $V\left(\ln \ell_{t}-\ln \ell_{t-1}\right)=V\left(\ln \alpha_{t}-\ln \alpha_{t-1}+\ln \hat{q}_{t}-\ln \hat{q}_{t-1}\right)$. Abstracting from the correlation between $x_{I-1}$ and $\alpha_{t-1}$, we can write the variance of log employment changes as a function of the variance of the changes in the endogenous productivity $\hat{q}_{t}$ and that of changes in the exogenous productivity $\alpha_{t}$. Using the $\operatorname{AR}(1)$ assumption, we get

$$
V\left(\ln \ell_{t}-\ln \ell_{t-1}\right)=\frac{2(1-\varphi)}{1-\varphi^{2}} \sigma_{u}^{2}+V\left(\ln \hat{q}_{t}-\ln \hat{q}_{t-1}\right) .
$$

The covariance of log employment changes can be written as a function of the variance of $\alpha$ and the persistence parameter:

$$
\operatorname{Cov}\left(\ln \ell_{t}-\ln \ell_{t-1}, \ln \ell_{t-1}-\ln \ell_{t-2}\right)=-\frac{(1-\varphi)^{2}}{1-\varphi^{2}} \sigma_{u}^{2} .
$$

Given the variance of endogenous productivity $V\left(\ln \hat{q}_{t}-\ln \hat{q}_{t-1}\right)$, we can infer the variance of the innovation $\sigma_{u}^{2}$ and the persistence parameter $\varphi$ from these two statistics. Similarly, when $\alpha$ follows a Markov chain, the variance and the covariance of log employment changes can be used to infer the size of the shock $\varepsilon$ and the persistence parameter $\rho$. The full calibration is reported in Table 1 and the comparison with the models targets are given in Table 2 .

\section{E.1.2 Data}

We estimate the variance and covariance of annual log employment changes using US census microdata from the Longitudinal Business Database (LBD). The LBD is an exhaustive establishment-level dataset that covers nearly all the non-farm private economy. The dataset provides longitudinally linked data on employment and payroll data for 21 million establishments over 1976-2000. The dataset is constructed using information from the business register, economic censuses, and surveys. ${ }^{4}$ We used the Synthetic LBD (U.S. Census Bureau, 2011), which is accessible through the virtual RDC. The results were then validated with the Census Bureau. We compute the variance and covariance of annual log employment change over the period 1976-2000 after excluding the three-digit SIC sectors 100 and 800-999. The estimated variance is 0.24 and the covariance is -0.05 .

\section{E.2 Extension 2: Small entrants and heterogeneous growth}

This section provides the details on the analysis of Section IV.A.

\footnotetext{
${ }^{4}$ For a detailed description of the dataset, see https://www.census.gov/ces/dataproducts/datasets/lbd.html.
} 
Table 1: Calibration of the two extensions

\begin{tabular}{|l|c|c|c|}
\hline & Parameter & Extension 1 & Extension 2 \\
\hline \hline Discount rate & $\beta$ & 0.947 & 0.947 \\
Disutility of labor & $\xi$ & 1.475 & 1.480 \\
Demand elasticity & $\psi$ & 0.2 & 0.2 \\
Innovation step: entrants & $\lambda_{E}$ & 1.50 & 1.50 \\
Innovation step: incumbents & $\lambda_{I}$ & 0.25 & 0.25 \\
Innovation cost curvature & $\gamma$ & 2.0 & 2.0 \\
Innovation cost: entrants & $\theta_{E}$ & 7.995 & 6.807 \\
Innovation cost: incumbents & $\theta_{I}$ & 1.333 & 1.134 \\
Entry cost & $\phi$ & 0.1642 & 0.0420 \\
Exogenous exit rate & $\delta$ & 0.001 & 0.015 \\
Transitory shock: size & $\varepsilon$ & 0.564 & 0.258 \\
Transitory shock: persistence & $\rho$ & 0.718 & - \\
Avg productivity from inactive lines & $h$ mean & 0.976 & 0.050 \\
Firing tax & $\tau$ & 0.000 & 0.000 \\
Weight function parameter & $\chi_{1}$ & - & 35.0 \\
Weight function parameter & $\chi_{2}$ & - & 26.5 \\
$\theta_{I}$ function parameter & $\chi_{3}$ & - & 0.4 \\
$\theta_{I}$ function parameter & $\chi_{4}$ & - & 0.8 \\
\hline
\end{tabular}

Table 2: Comparison between model outcome and the targets for the two extensions

\begin{tabular}{|l|r|rr|}
\hline & Data & \multicolumn{2}{|c|}{ Model } \\
& & Extension 1 & Extension 2 \\
\hline \hline Growth rate of output $g(\%)$ & 1.48 & 1.48 & 1.48 \\
Employment $L$ & 0.613 & 0.613 & 0.613 \\
Tail index $\kappa$ & 1.06 & 1.06 & - \\
Job-creation rate (\%) & 17.0 & - & 17.5 \\
Job-creation rate from entry (\%) & 6.4 & 6.4 & 6.7 \\
Entry rate (\%) & 12.6 & - & 6.6 \\
Variance of employment growth & 0.24 & 0.24 & - \\
Auto-cov. of employment growth & -0.05 & -0.05 & - \\
\hline
\end{tabular}

Note: The growth rate and employment targets are computed using BEA and BLS data; for the tail index, we use Axtell (2001) estimate; the job-flows data are computed from the Census Bureau BDS dataset, and the variance and autocovariance of employment growth are measured from LBD micro data. No statistics are reported when the statistics are not used as a target in the calibration. 


\section{E.2.1 Model setup}

The (normalized) value of a firm at the beginning of period is

$$
\hat{Z}(\hat{q}, \alpha, \ell)=(1-\delta) \hat{V}^{s}(\hat{q}, \alpha, \ell)+\delta \hat{V}^{o}(\ell),
$$

where

$$
\hat{V}^{o}(\ell)=-\tau \hat{w} \ell
$$

is the value of exit. The value of survival is

$$
\hat{V}^{s}(\hat{q}, \alpha, \ell)=\max _{\ell^{\prime} \geq 0, x_{I}}\left\{\hat{\Pi}\left(\hat{q}, \alpha, \ell, \ell^{\prime}, x_{I}\right)+\beta\left((1-u(\hat{q})) \hat{S}\left(x_{I}, \frac{\hat{q}}{1+g_{q}}, \ell^{\prime}\right)-u(\hat{q}) \tau \hat{w} \ell^{\prime}\right)\right\},
$$

where

$$
\hat{S}\left(x_{I}, \frac{\hat{q}}{1+g_{q}}, \ell^{\prime}\right)=\left(1-x_{I}\right) E_{\alpha^{\prime}}\left[\hat{Z}\left(\frac{\hat{q}}{1+g_{q}}, \alpha^{\prime}, \ell^{\prime}\right)\right]+x_{I} E_{\alpha^{\prime}}\left[\hat{Z}\left(\frac{\left(1+\lambda_{I}\right) \hat{q}}{1+g_{q}}, \alpha^{\prime}, \ell^{\prime}\right)\right] .
$$

The period profit is

$$
\hat{\Pi}\left(q, \alpha, \ell, \ell^{\prime}, x_{I}\right)=\left([\alpha \hat{q}]^{\psi} \ell^{\prime-\psi} \hat{Y}^{\psi}-\hat{w}\right) \ell^{\prime}-\theta_{I}(\hat{q}) \hat{q} x_{I}^{\gamma}-\tau \hat{w} \max \left\langle 0, \ell-\ell^{\prime}\right\rangle .
$$

For the entrants, the free entry condition is

$$
\max _{x_{E}}\left\{-\theta_{E} x_{E}^{\gamma}-\phi+\beta x_{E} \hat{\bar{V}}_{E}\right\}=0,
$$

where $x_{E}$ satisfies the optimality condition

$$
\beta \hat{\bar{V}}_{E}=\gamma \theta_{E} x_{E}^{\gamma-1}
$$

The expected benefit of entry, $\hat{\bar{V}}_{E}$, is now calculated from

$$
\hat{\bar{V}}_{E}=\int\left[\int \hat{Z}\left(\frac{\left(1+\lambda_{E}\right) \hat{q}}{1+g_{q}}, \alpha, 0\right)(N p(\hat{q})+(1-N) h(\hat{q})) d \hat{q}\right] \zeta(\alpha) d \alpha .
$$

\section{E.2.2 Transformed model and computation}

Define the frictionless level of employment without temporary shock as

$$
\ell^{*}(\hat{q} ; \hat{w}, \hat{Y}) \equiv \arg \max _{\ell^{\prime}}\left([\alpha \hat{q}]^{\psi} \ell^{\prime-\psi} \hat{Y}^{\psi}-\hat{w}\right) \ell^{\prime}
$$

with $\alpha=1$; that is,

$$
\ell^{*}(\hat{q} ; \hat{w}, \hat{Y})=\left(\frac{1-\psi}{\hat{w}}\right)^{\frac{1}{\psi}} \hat{q} \hat{Y}
$$

Also define $\Omega(\hat{w}, \hat{Y})$ by

$$
\Omega(\hat{w}, \hat{Y}) \equiv \frac{\ell^{*}(\hat{q} ; \hat{w}, \hat{Y})}{\hat{q}}
$$


In addition, define the deviation of employment from the frictionless level by

$$
\tilde{\ell} \equiv \frac{\ell}{\ell^{*}(\hat{q} ; \hat{w}, \hat{Y})} .
$$

Similarly, let

$$
\tilde{\ell}^{\prime} \equiv \frac{\ell^{\prime}}{\ell^{*}(\hat{q} ; \hat{w}, \hat{Y})}
$$

Then, the period profit can be rewritten as

$$
\begin{aligned}
& \hat{\Pi}\left(\hat{q}, \alpha, \ell, \ell^{\prime}, x_{I}\right)= \\
& \quad\left(\left[\frac{\alpha}{\Omega(\hat{w}, \hat{Y})}\right]^{\psi} \tilde{\ell}^{\prime-\psi} \hat{Y}^{\psi}-\hat{w}\right) \hat{q} \Omega(\hat{w}, \hat{Y}) \tilde{\ell}^{\prime}-\theta_{I}(\hat{q}) \hat{q} x_{I}^{\gamma}-\tau \hat{w} \max \left\langle 0, \hat{q} \Omega(\hat{w}, \hat{Y}) \tilde{\ell}-\hat{q} \Omega(\hat{w}, \hat{Y}) \tilde{\ell}^{\prime}\right\rangle .
\end{aligned}
$$

Thus this is linear in $\hat{q}$, and can be rewritten as $\hat{q} \tilde{\Pi}\left(\alpha, \tilde{\ell}, \tilde{\ell}^{\prime}, x_{I}\right)$, where

$$
\tilde{\Pi}\left(\alpha, \tilde{\ell}, \tilde{\ell}^{\prime}, x_{I}\right) \equiv\left(\left[\frac{\alpha}{\Omega(\hat{w}, \hat{Y})}\right]^{\psi} \tilde{\ell}^{\prime \prime \psi} \hat{Y}^{\psi}-\hat{w}\right) \Omega(\hat{w}, \hat{Y}) \tilde{\ell}^{\prime}-\theta_{I}(\hat{q}) x_{I}^{\gamma}-\tau \Omega(\hat{w}, \hat{Y}) \hat{w} \max \left\langle 0, \tilde{\ell}-\tilde{\ell}^{\prime}\right\rangle \text {. }
$$

Although the value function is not linear in $\hat{q}$, we still utilize the transformation on $\ell$ by defining the new value functions (abusing the ${ }^{\sim}$ notation on the value functions) as

$$
\tilde{Z}(\hat{q}, \alpha, \tilde{\ell})=(1-\delta) \tilde{V}^{s}(\hat{q}, \alpha, \tilde{\ell})+\delta \tilde{V}^{o}(\hat{q}, \tilde{\ell})
$$

where

$$
\begin{gathered}
\tilde{V}^{o}(\hat{q}, \tilde{\ell})=-\tau \hat{w} \hat{q} \Omega(\hat{w}, \hat{Y}) \tilde{\ell} \\
\tilde{V}^{s}(\hat{q}, \alpha, \tilde{\ell})=\max _{\tilde{\ell}^{\prime} \geq 0, x_{I}}\left\{\hat{q} \tilde{\Pi}\left(\alpha, \tilde{\ell}, \tilde{\ell}^{\prime}, x_{I}\right)+\beta\left((1-u(\hat{q})) \tilde{S}\left(x_{I}, \frac{\hat{q}}{1+g_{q}}, \tilde{\ell}^{\prime}\right)-u(\hat{q}) \tau \hat{w} \hat{q} \Omega(\hat{w}, \hat{Y}) \tilde{\ell^{\prime}}\right)\right\},
\end{gathered}
$$

where

$$
\tilde{S}\left(x_{I}, \frac{\hat{q}}{1+g_{q}}, \tilde{\ell}^{\prime}\right)=\left(1-x_{I}\right) E_{\alpha^{\prime}}\left[\tilde{Z}\left(\frac{\hat{q}}{1+g_{q}}, \alpha^{\prime},\left(1+g_{q}\right) \tilde{\ell}^{\prime}\right)\right]+x_{I} E_{\alpha^{\prime}}\left[\tilde{Z}\left(\frac{\left(1+\lambda_{I}\right) \hat{q}}{1+g_{q}}, \alpha^{\prime}, \frac{\left(1+g_{q}\right) \tilde{\ell^{\prime}}}{1+\lambda_{I}}\right)\right] \text {, }
$$

where the transformation of $\tilde{\ell}^{\prime}$ is similar to the baseline model.

For a given $\tilde{\ell}^{\prime}, x_{I}$ can be solved from the first-order condition

$$
\gamma \theta_{I}(\hat{q}) \hat{q} x_{I}^{\gamma-1}=\Gamma_{I},
$$

where

$$
\Gamma_{I} \equiv \beta(1-u(\hat{q}))\left\{E_{\alpha^{\prime}}\left[\tilde{Z}\left(\frac{\left(1+\lambda_{I}\right) \hat{q}}{1+g_{q}}, \alpha^{\prime}, \frac{\left(1+g_{q}\right) \tilde{\ell}^{\prime}}{1+\lambda_{I}}\right)\right]-E_{\alpha^{\prime}}\left[\tilde{Z}\left(\frac{\hat{q}}{1+g_{q}}, \alpha^{\prime},\left(1+g_{q}\right) \tilde{\ell}^{\prime}\right)\right]\right\} .
$$


The expected benefit of entry, $\hat{\bar{V}}_{E}$, is calculated with the same formula as above

$$
\hat{\bar{V}}_{E}=\int\left[\int \tilde{Z}\left(\frac{\left(1+\lambda_{E}\right) \hat{q}}{1+g_{q}}, \alpha, 0\right)(N p(\hat{q})+(1-N) h(\hat{q})) d \hat{q}\right] \zeta(\alpha) d \alpha,
$$

because $\tilde{\ell}=0$ is equivalent to $\ell=0$.

The computational steps are similar to the baseline model. The only difference is that we need to guess $\bar{f}(\hat{q})$ before performing the optimization. We update the guess at the same time as we update the aggregate variables. (It can also be done within the aggregate variables loop.) The following are the steps:

1. First, several variables can be computed from parameters. First, calculate $x_{E}^{*}$ from

$$
x_{E}^{*}=\left(\frac{\phi}{\theta_{E}(\gamma-1)}\right)^{\frac{1}{\gamma}} .
$$

2. Then $\hat{\bar{V}}_{E}$ can be computed from

$$
\hat{\bar{V}}_{E}=\frac{\gamma \theta_{E}}{\beta} x_{E}^{\gamma-1}
$$

3. Start the iteration. Guess $\hat{Y}, \hat{w}, m$, and $g$. Guess $\bar{f}(\hat{q})$.

Given $m$, we can calculate the value of $\mu$ by $\mu=X_{E}=m x_{E}^{*}$. From $\bar{f}(\hat{q})$ and $\mu$, we can obtain $u(\hat{q})$ and $p(\hat{q})$. (The value of $N$ can still be calculated by the same formula as in the baseline model.)

Now we are ready to solve the Bellman equation for the incumbents. We have two choice variables, $\tilde{\ell}^{\prime}$ and $x_{I}$. The first-order condition for $x_{I}$ is

$$
\gamma \theta_{I}(\hat{q}) \hat{q} x_{I}^{\gamma-1}=\Gamma_{I}
$$

and thus $x_{I}$ can be computed from

$$
x_{I}=\left(\frac{\Gamma_{I}}{\gamma \theta_{I}(\hat{q}) \hat{q}}\right)^{1 /(\gamma-1)}
$$

where

$\Gamma_{I} \equiv \beta(1-u(\hat{q}))\left\{E_{\alpha^{\prime}}\left[\tilde{Z}\left(\frac{\left(1+\lambda_{I}\right) \hat{q}}{1+g_{q}}, \alpha^{\prime}, \frac{\left(1+g_{q}\right) \tilde{\ell}^{\prime}}{1+\lambda_{I}}\right)\right]-E_{\alpha^{\prime}}\left[\tilde{Z}\left(\frac{\hat{q}}{1+g_{q}}, \alpha^{\prime},\left(1+g_{q}\right) \tilde{\ell}^{\prime}\right)\right]\right\}$.

We can see that $x_{I}$ is uniquely determined once we know $\tilde{\ell}^{\prime}$. Let the decision rule for $\tilde{\ell}^{\prime}$ be $\mathcal{L}^{\prime}(\hat{q}, \alpha, \tilde{\ell})$. Then $x_{I}=\mathcal{X}_{I}(\hat{q}, \alpha, \tilde{\ell})$.

4. Once all decision rules are computed, we can find $f(\hat{q}, \alpha, \tilde{\ell})$ by iterating over the density. 
5. Now, we check if the first guesses are consistent with the solution from the optimization. First, $\bar{f}(\hat{q})$ can be calculated from $f(\hat{q}, \alpha, \tilde{\ell})$.

The value $\hat{w}$ is checked from

$$
\hat{Y}=\left(\iiint[\alpha \hat{q}]^{\psi}\left[\ell^{*}(\hat{q} ; \hat{w}, \hat{Y}) \mathcal{L}^{\prime}(\hat{q}, \alpha, \tilde{\ell})\right]^{1-\psi} f(\hat{q}, \alpha, \tilde{\ell}) d \hat{q} d \alpha d \tilde{\ell}\right)^{\frac{1}{1-\psi}}
$$

The value of $\hat{Y}$ is checked from

$$
\frac{\hat{w}}{\hat{Y}-\hat{R}}=\xi
$$

where

$$
\hat{R}=\iiint \theta_{I} \hat{q} \mathcal{X}_{I}(\hat{q}, \alpha, \tilde{\ell})^{\gamma} f(\hat{q}, \alpha, \tilde{\ell}) d \hat{q} d \alpha d \tilde{\ell}+m\left(\phi+\theta_{E} x_{E}^{\gamma}\right) .
$$

To check the value of $g_{q}$, the condition $\frac{1}{N} \iiint \hat{q} f(\hat{q}, \alpha, \tilde{\ell}) d \alpha d \ell d q=1$ is used. Intuitively, when $g_{q}$ is too small, the stationary density $f(\hat{q}, \alpha, \tilde{\ell})$ implies the values of $\hat{q}$ that are too large.

To set $m$, we look at the free-entry condition. Because a large value of $m$ implies a large value of $\mu$, a larger value of $m$ implies a lower $\tilde{Z}$. Thus, the value of $m$ affects the computed value of $\hat{\bar{V}}_{E}$, through $\tilde{Z}$. Recall that

$$
\hat{\bar{V}}_{E}=\frac{\gamma \theta_{E}}{\beta} x_{E}^{\gamma-1}
$$

has to be satisfied, and this has to be equal to

$$
\hat{\bar{V}}_{E}=\int\left[\int \tilde{Z}\left(\frac{\left(1+\lambda_{E}\right) \hat{q}}{1+g_{q}}, \alpha, 0\right)(N p(\hat{q})+(1-N) h(\hat{q})) d \hat{q}\right] \zeta(\alpha) d \alpha .
$$

6. Go back to Step 3, until convergence.

\section{E.2.3 Calibration}

The overall calibration follows similar steps as the entrant-driven case in section III. The values of $\beta, \psi, \lambda_{I}, \gamma$, and $\delta$ are the same as in section III. For $\xi$, we target $L=0.61$ as in Section III. The values $\phi$ and $\varepsilon$ are set so that the model generates the overall job-creation rate and the job-creation rate by entrants close to the data. We assume $\lambda_{E}=1.50$. As in section III, the level parameter of incumbent innovation cost, now represented by $\bar{\theta}_{I}$ in equation (14), is set so that the overall growth rate of output, $g$, is $1.48 \%$. We set $\theta_{E}$ so that $\theta_{E} / \bar{\theta}_{I}=\lambda_{E} / \lambda_{I}$.

The new parameters of this extended model are $\chi_{1}$ and $\chi_{2}$ in equation (13) and $\chi_{3}$ and $\chi_{4}$ in equation (14). The value of $\chi_{1}$ is set as a large number so that the size of entrants becomes closer to the data. Given the job-creation rate from entrants, the size of entrants is reflected in the entry rate. A large value of $\chi_{1}$ makes the size of entrants small and thus increases the entry rate for a given job-creation rate by entrants. The 
Table 3: Size distribution; comparison between the US data and the model outcome

\begin{tabular}{|c|c|c|c|}
\hline & Data & Baseline & Extension \\
\hline $0-4$ & 0.495 & 0.917 & 0.491 \\
\hline $5-9$ & 0.223 & 0.017 & 0.253 \\
\hline $10-19$ & 0.138 & 0.020 & 0.135 \\
\hline $20-49$ & 0.089 & 0.025 & 0.087 \\
\hline 50-99 & 0.030 & 0.009 & 0.017 \\
\hline $100-249$ & 0.017 & 0.006 & 0.011 \\
\hline $250-499$ & 0.004 & 0.002 & 0.003 \\
\hline 500-999 & 0.002 & 0.001 & 0.001 \\
\hline $1000+$ & 0.001 & 0.001 & 0.001 \\
\hline
\end{tabular}

value of $\chi_{3}$ relates to the speed of growth by a small firm and thus is reflected in the size distribution of firms for small firms. The other two parameters, $\chi_{2}$ and $\chi_{4}$, also have effects on the size distribution of firms. Thus, these parameters are picked so that the size distribution of firms is close to the data. The parameter values are summarized in Table 1.

Table 3 compares the size distribution of firms in the data, the baseline model, and the extended model. The extended model is very close to the data.

Table 2 describes the outcomes of the models for $\tau=0$ in the baseline model and the extension. The discrepancy in the entry rate between the model and the data is substantially smaller in the extended model. Although it is not perfect, it seems to be the closest we can achieve given the functional forms. What is important here is that the results, and their intuitions, remain the same with these modifications that make the model outcome closer to the data.

\section{E.3 Robustness checks to the innovation size}

\section{E.3.1 Smaller innovation steps}

In the two calibrations in section III, the size of the incumbents' innovation step $\lambda_{I}$ is set at 0.25 , following estimates by Bena, Garlappi and Grüning (2015). In this section, we adopt an alternative strategy and use data on the establishment-level employment dynamics to calibrate this parameter. We set $\lambda_{I}$ to match the relative proportion of establishments creating jobs and destroying jobs. We measure the relative proportion of establishments creating and destroying jobs from the BLS annual Business Employment Dynamics Data and find a ratio of $1.05 . .^{5}$ The incumbents' innovation step is closely related to this statistic. For a given growth rate $g_{q}$, a smaller $\lambda_{I}$ implies a higher innovation probability $x_{I}$ and hence a larger proportion of establishments creating jobs. In fact, the

\footnotetext{
${ }^{5}$ We compute the average share of expanding establishments over the average share of contracting establishments over the available period (March 1994-March 2015). The data are publicly available at https://www.bls.gov/bdm/bdmann.htm.
} 
Table 4: Age distribution; comparison between the US data and the model outcome

\begin{tabular}{|l|r|r|r|}
\hline & Data & Baseline & Extension \\
\hline \hline 0 & 0.095 & 0.028 & 0.066 \\
1 & 0.076 & 0.027 & 0.059 \\
2 & 0.067 & 0.026 & 0.054 \\
3 & 0.060 & 0.025 & 0.050 \\
4 & 0.055 & 0.025 & 0.046 \\
5 & 0.050 & 0.024 & 0.043 \\
$6-10$ & 0.187 & 0.110 & 0.177 \\
$11-15$ & 0.123 & 0.096 & 0.129 \\
$16-20$ & 0.090 & 0.083 & 0.095 \\
$21-25$ & 0.066 & 0.072 & 0.071 \\
$26+$ & 0.130 & 0.484 & 0.211 \\
\hline
\end{tabular}

Note: The age distribution is computed from the US Census BDS dataset (average over 2003-2012). - age not available for all age classes before 2003. may not sum to one due to rounding.

Table 5: Exit rate by age; comparison between the US data and the model outcome

\begin{tabular}{|l|r|r|r|}
\hline & Data & Baseline & Extension \\
\hline \hline 1 & 0.227 & 0.028 & 0.109 \\
2 & 0.160 & 0.028 & 0.086 \\
3 & 0.138 & 0.028 & 0.076 \\
4 & 0.123 & 0.028 & 0.071 \\
5 & 0.115 & 0.028 & 0.068 \\
$6-10$ & 0.093 & 0.028 & 0.064 \\
$11-15$ & 0.073 & 0.028 & 0.060 \\
$16-20$ & 0.063 & 0.028 & 0.058 \\
$21-25$ & 0.057 & 0.028 & 0.057 \\
$26+$ & 0.048 & 0.028 & 0.057 \\
\hline
\end{tabular}

Note: The exit rate by age is computed from the US Census BDS dataset (average over 2003-2012).- age not available for all age classes before 2003. May not sum to one due to rounding. 
Table 6: Exit rate by size; comparison between the US data and the model outcome

\begin{tabular}{l|r|r|r|}
\hline & Data & Baseline & Extension \\
\hline \hline $0-4$ & 0.182 & 0.028 & 0.092 \\
$5-9$ & 0.047 & 0.028 & 0.041 \\
$10-19$ & 0.034 & 0.028 & 0.041 \\
$20-49$ & 0.028 & 0.028 & 0.041 \\
$50-99$ & 0.023 & 0.028 & 0.041 \\
$100-249$ & 0.018 & 0.028 & 0.041 \\
$250-499$ & 0.011 & 0.028 & 0.041 \\
$500-999$ & 0.009 & 0.028 & 0.041 \\
$1000+$ & 0.007 & 0.028 & 0.041 \\
\hline Note: The exit rate by size is computed from \\
the US Census BDS dataset (average over \\
1976-2012).
\end{tabular}

same growth rate can be reached either with a high $\lambda_{I}$ and low $x_{I}$, or with a low $\lambda_{I}$ and high $x_{I}$. To match the ratio of the relative proportion of establishments creating jobs, we set $\lambda_{I}$ at 0.0832 , which is lower than in the baseline model. For entrants, we first assume $\lambda_{E}=6 \lambda_{I}$, as in the entrant-driven growth calibration. The rest of the parameters are set following the same strategy as in section III. We then check the sensitivity of the results to assuming instead $\left(1+\lambda_{E}\right) /\left(1+\lambda_{I}\right)=2$, which also holds in the entrant-driven calibration in section III. In both cases, $\lambda_{E}$ is lower than in section III, though the difference is smaller when we assume $\left(1+\lambda_{E}\right) /\left(1+\lambda_{I}\right)=2$. The parameters and the targeted statistics are in Tables 7 and 8.

We report the results of this alternative calibration strategy in Table 9. We first consider the case in which $\lambda_{E} / \lambda_{I}$ is set at the same value as the entrant-driven baseline. As expected, with the lower innovation step $\lambda_{I}$, the incumbents' probability of innovating is higher than in the baseline model. On average, $48 \%$ of incumbents innovate in a given year compared with $8.4 \%$ in the baseline entrant-driven calibration. A large part of the results are qualitatively robust to this alternative calibration strategy: The firing tax leads to a decline in average productivity, to an increase in the innovation of incumbents, and to a reduction in the innovation of entrants. Quantitatively, the effects of the firing tax on the growth rate, however, differ from the entrant-driven baseline. We find the growth rate of aggregate productivity is virtually unaffected by the firing tax. This smaller negative effect of the firing tax on the growth rate comes from the smaller contribution of entrants to the growth rate. Although $\lambda_{E} / \lambda_{I}$ is the same as in section III, the contribution of entrants to the growth rate is lower. The decline in the entry rate therefore has a smaller impact on aggregate productivity growth. When we assume $\left(1+\lambda_{E}\right) /\left(1+\lambda_{I}\right)=2$, however, the contribution of entrants to the growth rate is closer to the baseline model, and we find the growth effect is also closer to the baseline model. The growth rate of output is reduced to $1.41 \%$, versus $1.39 \%$ in the baseline model, indicating that the relevant statistics for the contribution of entrants to growth and for the overall effect of firing costs on growth is $\left(1+\lambda_{E}\right) /\left(1+\lambda_{I}\right)$ rather than $\lambda_{E} / \lambda_{I}$. 
Table 7: Calibration: Alternative innovation sizes

\begin{tabular}{|l|c|c|c|c|}
\hline & Parameter & $\begin{array}{c}\text { Small } \lambda_{I} \\
\text { same } \lambda_{E} / \lambda_{I}\end{array}$ & $\begin{array}{c}\text { Small } \lambda_{I} \\
\text { same }\left(1+\lambda_{E}\right) /\left(1+\lambda_{I}\right)\end{array}$ & $\lambda_{I}=0$ \\
\hline \hline Discount rate & $\beta$ & 0.947 & 0.947 & 0.947 \\
Disutility of labor & $\xi$ & 1.482 & 1.476 & 1.496 \\
Demand elasticity & $\psi$ & 0.2 & 0.2 & 0.2 \\
Innovation step: entrants & $\lambda_{E}$ & 0.50 & 1.17 & 1.50 \\
Innovation step: incumbents & $\lambda_{I}$ & 0.08 & 0.08 & 0.00 \\
Innovation cost curvature & $\gamma$ & 2.0 & 2.0 & 2.0 \\
Innovation cost: entrants & $\theta_{E}$ & 0.417 & 0.239 & 5.750 \\
Innovation cost: incumbents & $\theta_{I}$ & 0.070 & 0.119 & 0.958 \\
Entry cost & $\phi$ & 0.8502 & 1.7034 & 0.1644 \\
Exogenous exit rate & $\delta$ & 0.00056 & 0.00084 & 0.00127 \\
Transitory shock: size & $\varepsilon$ & 0.260 & 0.271 & 0.223 \\
Avg productivity from inactive lines & $h$ mean & 0.976 & 0.976 & 0.976 \\
Firing tax & $\tau$ & 0.0 & 0.0 & 0.0 \\
\hline
\end{tabular}

\section{E.3.2 When only entrants innovate}

To evaluate the importance of including the incumbents' innovation in the model, we consider the case in which only entrants innovate. We set $\lambda_{I}=0$ and re-calibrate the parameters $\theta_{E}, \xi, \varepsilon$, and $\delta$ to match the growth rate of output per worker, the employment rate, the job-creation rate, and the tail index of the firm size distribution. The other parameters (including $\phi$ ) are kept identical to the calibration in section III. The parameters and the targeted statistics are in Tables 7 and 8 . Because entrants are the only innovators, we can no longer match the job-creation rate by entrants, because $\theta_{E}$ needs to be set at a value that is consistent with the growth rate. The results, reported in Table 9, show that ignoring the incumbents' innovation would lead to overestimating the decline in the growth rate. When only entrants innovate, the positive impact on the incumbents' innovation is absent and the firing tax therefore leads to a larger decline in innovation and aggregate productivity growth. The effect is quantitatively substantial. We find that the growth rate in the economy with firing costs is $1.33 \%$, versus $1.39 \%$ in our entrant-driven growth baseline.

\section{E.4 The effect of labor taxes and innovation subsidies}

Our model can easily be extended to analyze the effects of taxes and subsidies. Here, we consider a labor tax of the rate $\eta \in[0,1]$ and $\mathrm{R} \& \mathrm{D}$ subsidies at the rate $s \in[0,1]$ to both incumbents and entrants.

The budget constraint for the consumer changes to

$$
A_{t+1}+C_{t}=\left(1+r_{t}\right) A_{t}+(1-\eta) w_{t} L_{t}+T_{t} .
$$

This changes the first-order condition for the consumer to

$$
\frac{w_{t}}{C_{t}}=\frac{\xi}{1-\eta} \text {. }
$$


Table 8: Comparison between model outcome and the targets for the alternative calibrations

\begin{tabular}{|l|r|rrr|}
\hline & Data & Small $\lambda_{I}$ & Small $\lambda_{I}$ & $\lambda_{I}=0$ \\
& & same $\lambda_{E} / \lambda_{I}$ & same $\left(1+\lambda_{E}\right) /\left(1+\lambda_{I}\right)$ & \\
\hline \hline Growth rate of output $g(\%)$ & 1.48 & 1.48 & 1.48 & 1.48 \\
Employment $L$ & 0.613 & 0.613 & 0.613 & 0.613 \\
Tail index $\kappa$ & 1.06 & 1.06 & 1.06 & 1.06 \\
Job-creation rate (\%) & 17.0 & 17.0 & 17.0 & 17.0 \\
Job-creation rate from entry (\%) & 6.4 & 6.4 & 6.4 & - \\
Positive employment growth & 1.05 & 1.04 & - & - \\
\hline
\end{tabular}

Note: The growth rate and employment targets are computed using BEA and BLS data; for the tail index, we use Axtell (2001) estimate; the job-flows data are computed from the Census Bureau BDS dataset and the variance and autocovariance of employment growth are measured from LBD micro data. "Positive employment growth" refers to the ratio of expanding private sector establishments over contracting establishments computed from the BLS BED dataset. No statistics are reported when the statistics are not used as a target in the calibration.

Table 9: Robustness: Smaller innovation steps

\begin{tabular}{|l|rr|rr|rr|}
\hline & \multicolumn{2}{|c|}{ Small $\lambda_{I}$} & \multicolumn{2}{|c|}{ Small $\lambda_{I}$} & \multicolumn{2}{c|}{$\lambda_{I}=0$} \\
& \multicolumn{2}{|c|}{ same $\lambda_{E} / \lambda_{I}$} & same $\left(1+\lambda_{E}\right) /\left(1+\lambda_{I}\right)$ & & \\
& $\tau=0.0$ & $\tau=0.3$ & $\tau=0.0$ & $\tau=0.3$ & $\tau=0.0$ & $\tau=0.3$ \\
\hline \hline Growth rate of output $g(\%)$ & 1.48 & 1.49 & 1.48 & 1.41 & 1.48 & 1.33 \\
Innovation probability: incumbents $\bar{x}_{I}$ & 0.483 & 0.528 & 0.303 & 0.333 & 0.000 & 0.000 \\
Innovation probability: entrants $x_{E}$ & 1.000 & 1.000 & 1.000 & 1.000 & 0.169 & 0.169 \\
Creative-destruction rate $\mu(\%)$ & 4.50 & 3.83 & 3.09 & 2.64 & 4.00 & 3.57 \\
Employment $L$ & 100 & 98.8 & 100 & 98.8 & 100 & 98.2 \\
Normalized output $\hat{Y}$ & 100 & 98.0 & 100 & 98.1 & 100 & 97.7 \\
Normalized average productivity $\hat{Y} / L$ & 100 & 99.4 & 100 & 99.3 & 100 & 99.5 \\
Number of active products $N$ & 0.988 & 0.986 & 0.974 & 0.969 & 0.969 & 0.966 \\
Job-creation rate (\%) & 17.0 & 5.0 & 17.0 & 4.6 & 17.0 & 6.8 \\
Job-creation rate from entry $(\%)$ & 6.4 & 4.4 & 6.4 & 4.2 & 9.6 & 6.6 \\
Job-destruction rate $(\%)$ & 17.0 & 5.0 & 17.0 & 4.6 & 17.0 & 6.8 \\
Job-destruction rate from exit $(\%)$ & 4.9 & 3.9 & 3.2 & 2.7 & 4.1 & 3.7 \\
R\&D ratio $R / Y(\%)$ & 12.0 & 11.3 & 11.6 & 10.7 & 12.8 & 11.7 \\
\hline
\end{tabular}

Note: $L, \hat{Y}$, and $\hat{Y} / L$ are set at 100 in the $\tau=0.0$ case. 
The other equilibrium conditions are unchanged. Because $\xi$ is endogenously targeted in the calibration, the baseline model is identical even when the value of $\eta$ is nonzero. When the value of $\xi$ is $\xi_{0}$ when $\eta=0$, the equilibrium is identical when $\xi$ is set at $\xi_{0}(1-\eta)$ in the economy with $\eta>0$. Moreover, the experiment starting from $\eta=\eta_{0}$ to $\eta=\eta_{1}$ is equivalent to the experiment starting from $\eta=0$ to $\eta=1-\left(1-\eta_{1}\right) /\left(1-\eta_{0}\right)$. For example, an experiment of changing $\eta=0.3$ to $\eta=0.5$ is the same as starting from $\eta=0$ to $\eta=1-(1-0.5) /(1-0.3)=0.286$. Changing $\eta=0.3$ to $\eta=0.35$ is the same as starting from $\eta=0$ to $\eta=1-(1-0.35) /(1-0.3)=0.071$.

To facilitate the comparison to the literature, we also consider a more general form of preferences. Following Rogerson and Wallenius (2009), we specify the period utility as

$$
\log \left(C_{t}\right)-\xi \frac{L_{t}^{1+\nu}}{1+\nu}
$$

where $\nu \geq 0$. Our baseline model is the special case of $\nu=0 .{ }^{6}$ For this utility function, only change that is necessary for the equilibrium conditions is the optimality condition for the labor-leisure choice. Instead of (2), the first-order condition is

$$
\frac{w_{t}}{C_{t}}=\frac{\xi L_{t}^{\nu}}{1-\eta}
$$

The analytical characterization of the model (Appendix B) is almost identical, except that the labor-market equilibrium condition must be modified to

$$
\frac{\hat{w}(m)}{\hat{Y}(m, L)-\hat{R}(m, L)}=\frac{\xi L^{\nu}}{1-\eta} .
$$

The computation of the model (Appendix D) is also similar, except that step 6 (b) uses the consumption value of $\hat{C}=L^{-\nu} \hat{w} / \xi$.

First, we compare, in the entrant-driven growth economy, the effects of the firing tax with that of a $5 \%$ labor $\operatorname{tax}(\eta=0.05)$. The baseline model is $\eta=0$. We report the results for different values of the Frisch elasticity parameter $\nu$ in Table 10. In our baseline model $(\nu=0)$, we find the labor tax reduces the growth rate to $1.38 \%$, whereas the firing tax reduces the growth rate to $1.39 \%$. This finding shows the effect of the firing tax in the entrant-driven case in section III is of the same magnitude as a $5 \%$ labor tax.

Next, we make a comparison to the previous study by Rogerson and Wallenius (2009). To be consistent with their study (they consider the $30 \%$ case as the US and the $50 \%$ case as the continental Europe), we start from $\eta=0.30$ and recalibrate. Then we compare the outcome with the case of $\eta=0.50$.

\footnotetext{
${ }^{6}$ Ohanian, Raffo and Rogerson (2008) consider a similar utility function of the form (omitting the subsistence consumption and government consumption)

$$
\alpha \log \left(C_{t}\right)+(1-\alpha) \frac{\left(\bar{L}-L_{t}\right)^{1-\gamma}-1}{1-\gamma},
$$

where $\alpha \in(0,1), \gamma \geq 0$, and $\bar{L}>0$, in our notation. Note that our baseline model corresponds to the case with $\gamma=0$. Their overall conclusion is that a neoclassical model with this form of utility (with subsistence consumption) with changes in taxes explain the post-war change in hours across OECD countries. They note that the results are robust to the values of $\gamma \in[0,2]$.
} 
Table 10: Labor tax (5\%) and the labor-supply elasticity

\begin{tabular}{|c|c|c|c|c|c|}
\hline & \multirow[t]{2}{*}{$\eta=0.0$} & \multicolumn{4}{|c|}{$\eta=0.05$} \\
\hline & & $\nu=0.0$ & $\nu=0.5$ & $\nu=2$ & $\nu=10$ \\
\hline Growth rate of output $g(\%)$ & 1.48 & 1.38 & 1.42 & 1.45 & 1.47 \\
\hline Innovation probability: incumbents $\bar{x}_{I}$ & 0.084 & 0.084 & 0.084 & 0.084 & 0.084 \\
\hline Innovation probability: entrants $x_{E}$ & 0.143 & 0.143 & 0.143 & 0.143 & 0.143 \\
\hline Creative-destruction rate $\mu(\%)$ & 2.65 & 2.37 & 2.47 & 2.56 & 2.63 \\
\hline Employment $L$ & 100 & 94.5 & 96.4 & 98.3 & 99.5 \\
\hline Normalized output $\hat{Y}$ & 100 & 94.4 & 96.4 & 98.2 & 99.5 \\
\hline Normalized average productivity $\hat{Y} / L$ & 100 & 99.9 & 99.9 & 100 & 100.0 \\
\hline Number of active products $N$ & 0.964 & 0.959 & 0.961 & 0.962 & 0.963 \\
\hline Job-creation rate $(\%)$ & 17.0 & 16.6 & 16.7 & 16.9 & 17.0 \\
\hline Job-creation rate from entry $(\%)$ & 6.4 & 5.8 & 6.0 & 6.2 & 6.4 \\
\hline Job-destruction rate $(\%)$ & 17.0 & 16.6 & 16.7 & 16.9 & 17.0 \\
\hline Job-destruction rate from exit (\%) & 2.8 & 2.5 & 2.6 & 2.7 & 2.7 \\
\hline $\mathrm{R} \& \mathrm{D}$ ratio $R / Y$ & 11.5 & 11.1 & 11.2 & 11.4 & 11.5 \\
\hline
\end{tabular}

Note: $L, \hat{Y}$, and $\hat{Y} / L$ are set at 100 in the $\eta=0.0$ case.

Table 11: Effect of a large increase in the labor tax (0.30 to 0.50$)$

\begin{tabular}{|l|c|c|c|c|c|}
\hline & \multirow{2}{*}{$\eta=0.30$} & \multicolumn{4}{|c|}{$\eta=0.50$} \\
\cline { 3 - 6 } & & $\nu=0$ & $\nu=0.5$ & $\nu=2$ & $\nu=10$ \\
\hline \hline Growth rate of output $g(\%)$ & 1.48 & 0.95 & 1.11 & 1.29 & 1.43 \\
Innovation probability: incumbents $\bar{x}_{I}$ & 0.084 & 0.085 & 0.085 & 0.084 & 0.084 \\
Innovation probability: entrants $x_{E}$ & 0.143 & 0.143 & 0.143 & 0.143 & 0.143 \\
Creative-destruction rate $\mu(\%)$ & 2.65 & 1.09 & 1.56 & 2.09 & 2.50 \\
Employment $L$ & 100 & 68.8 & 78.7 & 89.1 & 97.0 \\
Normalized output $\hat{Y}$ & 100 & 68.0 & 78.2 & 88.9 & 96.9 \\
Normalized average productivity $\hat{Y} / L$ & 100 & 98.7 & 99.4 & 99.7 & 99.9 \\
Number of active products $N$ & 0.964 & 0.916 & 0.940 & 0.954 & 0.961 \\
Job-creation rate (\%) & 17.0 & 14.5 & 15.3 & 16.1 & 16.8 \\
Job-creation rate from entry $(\%)$ & 6.4 & 2.8 & 3.9 & 5.1 & 6.1 \\
Job-destruction rate (\%) & 17.0 & 14.5 & 15.3 & 16.1 & 16.8 \\
Job-destruction rate from exit $(\%)$ & 2.8 & 1.2 & 1.7 & 2.2 & 2.6 \\
R\&D ratio $R / Y$ & 11.5 & 8.2 & 9.44 & 10.6 & 11.3 \\
\hline
\end{tabular}

Note: $L, \hat{Y}$, and $\hat{Y} / L$ are set at 100 in the $\eta=0.30$ case. 
Table 12: Firing tax and the labor supply elasticity

\begin{tabular}{|l|c|c|c|c|c|}
\hline & $\tau=0.0$ & \multicolumn{4}{|c|}{$\tau=0.3$} \\
\cline { 3 - 6 } & & $\nu=0.0$ & $\nu=0.5$ & $\nu=2$ & $\nu=10$ \\
\hline \hline Growth rate of output $g(\%)$ & 1.48 & 1.39 & 1.40 & 1.40 & 1.41 \\
Innovation probability: incumbents $\bar{x}_{I}$ & 0.084 & 0.091 & 0.091 & 0.091 & 0.091 \\
Innovation probability: entrants $x_{E}$ & 0.143 & 0.143 & 0.143 & 0.143 & 0.143 \\
Creative-destruction rate $\mu(\%)$ & 2.65 & 2.30 & 2.31 & 2.33 & 2.34 \\
Employment $L$ & 100 & 98.8 & 99.2 & 99.6 & 99.9 \\
Normalized output $\hat{Y}$ & 100 & 98.1 & 98.5 & 98.9 & 99.2 \\
Normalized average productivity $\hat{Y} / L$ & 100 & 99.3 & 99.3 & 99.3 & 99.3 \\
Number of active products $N$ & 0.964 & 0.958 & 0.959 & 0.959 & 0.959 \\
Job-creation rate (\%) & 17.0 & 4.7 & 4.8 & 4.8 & 4.8 \\
Job-creation rate from entry $(\%)$ & 6.4 & 4.3 & 4.3 & 4.3 & 4.4 \\
Job-destruction rate (\%) & 17.0 & 4.7 & 4.8 & 4.8 & 4.8 \\
Job-destruction rate from exit $(\%)$ & 2.8 & 2.4 & 2.4 & 2.4 & 2.4 \\
R\&D ratio $R / Y$ & 11.5 & 10.6 & 10.6 & 10.6 & 10.6 \\
\hline
\end{tabular}

Note: $L, \hat{Y}$, and $\hat{Y} / L$ are set at 100 in the $\tau=0.0$ case.

We report the results in Table 11. We find the employment rate is reduced by $31 \%$ for our $\nu=0$ calibration. We find that when we set $\nu$ to the same value as Rogerson and Wallenius (2009) $(\nu=0.50)$, labor declines by $21.3 \%$, which is similar to Rogerson and Wallenius's $22 \%$ decline. This similarity is not trivial, because Rogerson and Wallenius (2009) consider a substantially richer labor-supply model (with life-cycle heterogeneity, extensive and intensive margin of labor supply).

We find the large increase in the labor tax reduces the growth rate from $1.48 \%$ to $0.95 \%$ for $\nu=0$, and to $1.11 \%$ for $\nu=0.5$. As we discussed in the main text, for the firing tax, the decline in the growth rate is a result of two opposite effects for incumbents and entrants. In the case of the labor tax, entry is reduced because the labor tax reduces profitability, similarly to the firing-tax case. The incumbents' innovation is higher here, too, but note the mechanism is not the same as in the case of the firing tax. The taxescaping effect that we highlight in the case of the firing tax is absent in the case of the labor tax because innovating would not affect the tax rate. The incumbent innovation increases because entrants' innovation decreases (what we call the "creative-destruction effect" in the main text).

For completeness, we repeat the effects of the firing tax for different values of $\nu$. The results are reported in Table 12. We find the effect of the firing tax when $\nu=0.5$ is virtually identical to the $\nu=0.0$ case. When $\nu$ is higher, a high firing tax leads to a smaller decline in the growth rate than when $\nu$ is lower, because the entry rate decreases less. The overall growth effects, however, are similar across different values of $\nu$.

Now we consider the R\&D subsidy. The R\&D subsidy changes the cost for innovation for incumbents and entrants. In particular, the innovation cost for the incumbents is

$$
\mathbf{r}_{I j t}=(1-s) \theta_{I} Q_{t} \frac{q_{j t}}{\bar{q}_{t}} x_{I j t}^{\gamma}
$$


and the innovation cost for the entrant is

$$
\mathbf{r}_{E j t}=(1-s) \theta_{E} Q_{t} x_{E j t}^{\gamma} .
$$

In the analytical characterization (Appendix B), this modification does not alter the calculation of $\hat{R}$ in (7), because the resource cost does not change with subsidies. The equilibrium conditions change in the incumbent's innovation choice and the entrants' choices. The value functions for incumbents are now

$$
\hat{Z}(\hat{q}, \alpha)=(1-\delta) \hat{V}^{s}(\hat{q}, \alpha)
$$

where

$$
\hat{V}^{s}(\hat{q}, \alpha)=\max _{x_{I}} \psi \alpha \hat{q} \frac{\hat{Y}}{N}-(1-s) \theta_{I} \hat{q} x_{I}^{\gamma}+\beta(1-\mu) \hat{S}\left(x_{I}, \hat{q} /\left(1+g_{q}\right)\right)
$$

and

$\hat{S}\left(x_{I}, \hat{q} /\left(1+g_{q}\right)\right)=\left(1-x_{I}\right) \int \hat{Z}\left(\hat{q} /\left(1+g_{q}\right), \alpha^{\prime}\right) \zeta\left(\alpha^{\prime}\right) d \alpha^{\prime}+x_{I} \int \hat{Z}\left(\left(1+\lambda_{I}\right) \hat{q} /\left(1+g_{q}\right), \alpha^{\prime}\right) \zeta\left(\alpha^{\prime}\right) d \alpha^{\prime}$.

Similar to the baseline model, the value function is linear:

$$
\hat{Z}(\hat{q}, \alpha)=\mathcal{A} \alpha \hat{q}+\mathcal{B} \hat{q}
$$

The optimal $x_{I}$ is

$$
x_{I}=\left(\frac{\beta(1-\mu) \lambda_{I}(\mathcal{A}+\mathcal{B})}{\left(1+g_{q}\right) \gamma(1-s) \theta_{I}}\right)^{\frac{1}{\gamma-1}}
$$

and the constants are

$$
\mathcal{A}=(1-\delta) \psi \frac{\hat{Y}}{N}
$$

and $\mathcal{B}$ solves

$$
\mathcal{B}=(1-\delta) \beta(1-\mu)\left(1+\frac{\gamma-1}{\gamma} \lambda_{I} x_{I}\right) \frac{\mathcal{A}+\mathcal{B}}{1+g_{q}} .
$$

The change from the baseline model is therefore the expression for $x_{I}$ only. For entrants, the optimal innovation rate for the potential entrant is now

$$
x_{E}^{*}=\left(\frac{\phi}{(1-s) \theta_{E}(\gamma-1)}\right)^{\frac{1}{\gamma}},
$$

and the free-entry condition (8) must be changed to

$$
\frac{\gamma(1-s) \theta_{E} x_{E}^{\gamma-1}}{\beta}=\hat{\bar{V}}_{E}
$$

The computation of the model (Appendix D) would change in a few places. First, in step 2, the entrants' innovation equations are

$$
x_{E}^{*}=\left(\frac{\phi}{(1-s) \theta_{E}(\gamma-1)}\right)^{\frac{1}{\gamma}}
$$


Table 13: Innovation subsidies

\begin{tabular}{|l|r|r|}
\hline & Baseline, $\tau=0.0$ & $\tau=0.3$ \\
& $s=0.0$ & $s=0.073$ \\
\hline \hline Growth rate of output $g(\%)$ & 1.48 & 1.48 \\
Innovation probability: incumbents $\bar{x}_{I}$ & 0.084 & 0.094 \\
Innovation probability: entrants $x_{E}$ & 0.143 & 0.149 \\
Creative-destruction rate $\mu(\%)$ & 2.65 & 2.50 \\
Employment $L$ & 100 & 99.7 \\
Normalized output $\hat{Y}$ & 100 & 99.0 \\
Normalized average productivity $\hat{Y} / L$ & 100 & 99.3 \\
Number of active products $N$ & 0.964 & 0.961 \\
Job-creation rate (\%) & 17.0 & 5.1 \\
Job-creation rate from entry $(\%)$ & 6.4 & 4.6 \\
Job-destruction rate $(\%)$ & 17.0 & 5.1 \\
Job-destruction rate from exit $(\%)$ & 2.8 & 2.6 \\
R\&D ratio $R / Y$ & 11.5 & 11.4 \\
\hline
\end{tabular}

Note: $L, \hat{Y}$, and $\hat{Y} / L$ are set at 100 in the $\tau=0.0$ and $s=0.0$ case.

and

$$
\hat{\bar{V}}_{E}=\frac{(1-s) \gamma \theta_{E}}{\beta} x_{E}^{\gamma-1} .
$$

Second, in step 4 , because now the flow profit is

$\tilde{\Pi}\left(\alpha, \tilde{\ell}, \tilde{\ell}^{\prime}, x_{I}\right) \equiv\left(\left[\frac{\alpha}{\Omega(\hat{w}, \hat{Y})}\right]^{\psi} \tilde{\ell}^{\prime-\psi} \hat{Y}^{\psi}-\hat{w}\right) \Omega(\hat{w}, \hat{Y}) \tilde{\ell}^{\prime}-(1-s) \theta_{I} x_{I}^{\gamma}-\tau \Omega(\hat{w}, \hat{Y}) \hat{w} \max \left\langle 0, \tilde{\ell}-\tilde{\ell}^{\prime}\right\rangle$,

with $\Omega(\hat{w}, \hat{Y}) \equiv \ell^{*}(\hat{q} ; \hat{w}, \hat{Y}) / \hat{q}$, the first-order condition for $x_{I}$ is

$$
\gamma(1-s) \theta_{I} x_{I}^{\gamma-1}=\Gamma_{I}
$$

and thus $x_{I}$ can be computed from

$$
x_{I}=\left(\frac{\Gamma_{I}}{\gamma(1-s) \theta_{I}}\right)^{1 /(\gamma-1)},
$$

where $\Gamma_{I} \equiv \beta(1-\mu) E_{\alpha^{\prime}}\left[\tilde{Z}\left(\alpha^{\prime},\left(1+g_{q}\right) \tilde{\ell}^{\prime} /\left(1+\lambda_{I}\right)\right)\left(1+\lambda_{I}\right)-\tilde{Z}\left(\alpha^{\prime},\left(1+g_{q}\right) \tilde{\ell}^{\prime}\right)\right] /\left(1+g_{q}\right)$. Third, in step $6(\mathrm{~b})$, the free-entry condition is once again

$$
\hat{\bar{V}}_{E}=\frac{\gamma(1-s) \theta_{E}}{\beta} x_{E}^{\gamma-1} .
$$

The results are in Table 13. We find we would need a subsidy equal to $7.3 \%$ to offset the effect of the firing tax on the growth rate. 


\section{E.5 Expanding-variety model}

Our baseline model is a quality-ladder model. There are alternative formulations of innovation in the literature, and seeing how different formulations of innovation can affect the outcome of the model is of interest. Here, we consider the expanding-variety model of Romer (1990). While we find some overlap in the notation with the main text, we intend this section to be self-contained.

As in the standard expanding-variety model, we assume innovation is conducted only by the entrants. For simplicity, we assume no exogenous exit of firms occurs. Furthermore, we assume no exogenous productivity shocks occur, which implies the model has a limited ability to match observed job flows. In the model, incumbents do not contribute to job creation, and job destruction is uniform across incumbents; and therefore, job flows will be lower than in the data. By contrast, with innovation conducted by the incumbents, the quality-ladder model naturally generates both job creation and job destruction by incumbents even without exogenous shocks. Although we could easily add exogenous shocks to the model to better fit the job-flows data, we choose here to consider the simplest version of the model to study the main mechanism through which firing taxes affect growth.

The production structure is similar to our baseline model. The final goods, which are used both for consumption and $\mathrm{R} \& \mathrm{D}$, are produced using only intermediate goods, and the differentiated intermediate goods are produced by monopolists using labor. Let the final goods produced at time $t$ be $Y_{t}$. The quantity of intermediate good $j$ used at time $t$ is denoted $y_{j t}$. The final-goods production function is

$$
Y_{t}=\left(\int_{0}^{N_{t}} y_{j t}^{1-\psi} d j\right)^{\frac{1}{1-\psi}}
$$

where $1 / \psi$ is the elasticity of substitution across goods, and $N_{t}$ is the number of goods produced at time $t$. The final-goods market is perfectly competitive. After the cost minimization by the final-goods producers, the inverse demand function for intermediate $\operatorname{good} j$ is

$$
p_{j t}=y_{j t}^{-\psi} Y_{t}^{\psi}
$$

where $p_{j t}$ is the price of good $j$ at time $t$.

Each entrant needs to pay $\eta^{-1} N_{t}^{\frac{\frac{\psi}{1-\psi}}{1-1}}$ units of final goods to come up with a new variety and enter. The free-entry condition for innovation equates the value of innovation, $\mathbf{V}_{t}$, to the cost. Thus,

$$
\mathbf{V}_{t}=\eta^{-1} N_{t}^{\frac{\psi}{1-\psi}-1}
$$

The growth in the number of variety depends on aggregate spending by entrants on developing new varieties $R_{t}$ :

$$
N_{t+1}-N_{t}=\eta N_{t}^{1-\frac{\psi}{1-\psi}} R_{t}
$$

On the consumer side, the preferences are assumed to be the same as in the baseline model:

$$
\mathbf{U}=\sum_{t=0}^{\infty} \beta^{t}\left[\log \left(C_{t}\right)-\xi L_{t}\right]
$$


where $C_{t}$ is the final goods consumption and $L_{t}$ is labor supply.

Let us focus on the balanced-growth path. Let the growth rate of $N_{t}$ be $g$, that is $\left(N_{t+1}-N_{t}\right) / N_{t}=g$. Along the balanced-growth path, $Y_{t}, C_{t}, R_{t}$, and $w_{t}$ grow at the rate $g_{Y}$, where

$$
1+g_{Y}=(1+g)^{\frac{\psi}{1-\psi}}
$$

From the Euler equation of the consumer, the firm discounts future profits at rate $\beta\left(C_{t} / C_{t+1}\right)=\beta /\left(1+g_{Y}\right)$. Thus, the Bellman equation for the intermediate good producer is

$$
V_{t}\left(\ell_{t-1}\right)=\max _{\ell_{t}} \ell_{t}^{1-\psi} Y_{t}^{\psi}-w_{t} \ell_{t}-\tau w_{t} \max \left\langle 0, \ell_{t-1}-\ell_{t}\right\rangle+\frac{\beta}{1+g_{Y}} V_{t+1}\left(\ell_{t}\right) .
$$

As in the quality-ladder model, we can make the Bellman equation stationary. Here, we normalize by dividing $Y_{t}, C_{t}, R_{t}$, and $w_{t}$ by $N_{t}^{\frac{\psi}{1-\psi}}$ and dividing $\ell_{t}$ by $N_{t}^{-1}$. The value function $V_{t}\left(\ell_{t-1}\right)$ is divided by $N_{t}^{\frac{\psi}{1-\psi}-1}$ :

$$
\hat{V}_{t}\left(\hat{\ell}_{t-1}\right)=\max _{\hat{\ell}_{t}} \hat{\ell}_{t}^{1-\psi} \hat{Y}_{t}^{\psi}-\hat{w}_{t} \hat{\ell}_{t}-\tau \hat{w}_{t} \max \left\langle 0,(1+g) \hat{\ell}_{t-1}-\hat{\ell}_{t}\right\rangle+\frac{\beta}{1+g} \hat{V}_{t+1}\left(\hat{\ell}_{t}\right) .
$$

Note that we used $\ell_{t-1} / N_{t}^{-1}=(1+g) \ell_{t-1} / N_{t-1}^{-1}=(1+g) \hat{\ell}_{t-1}$ and $V_{t+1}\left(\hat{\ell}_{t}\right) / N_{t}^{\frac{\psi}{1-\psi}-1}=$ $(1+g)^{\frac{\psi}{1-\psi}-1} V_{t+1}\left(\hat{\ell}_{t}\right) / N_{t+1}^{\frac{\psi}{1-\psi}-1}=\left(1+g_{Y}\right)(1+g)^{-1} \hat{V}_{t+1}\left(\hat{\ell}_{t}\right)$. This can be written without the time subscript as

$$
\hat{V}(\hat{\ell})=\max _{\hat{\ell}^{\prime}} \hat{\ell}^{1-\psi} \hat{Y}^{\psi}-\hat{w} \hat{\ell}^{\prime}-\tau \hat{w} \max \left\langle 0,(1+g) \hat{\ell}-\hat{\ell}^{\prime}\right\rangle+\frac{\beta}{1+g} \hat{V}\left(\hat{\ell}^{\prime}\right) .
$$

Note that because all the entrants are identical, and the employment decision only depends on past employment $\hat{\ell}$, the employment process is deterministic and identical across firms: Firms of the same age have the same employment level.

\section{E.5.1 No-firing-tax case}

With no firing tax, the intermediate good firm's problem becomes static. The maximization problem yields the optimal $\ell_{t}$ as

$$
\ell_{t}^{*}=\left(\frac{1-\psi}{w_{t}}\right)^{\frac{1}{\psi}} Y_{t}=\left(\frac{1-\psi}{\hat{w}}\right)^{\frac{1}{\psi}} \hat{Y} N_{t}^{-1} .
$$

In the context of the normalized Bellman, equation (15), $\hat{\ell}^{\prime}$ is thus $((1-\psi) / \hat{w})^{\frac{1}{\psi}} \hat{Y}$, and therefore the value function satisfies ( $\hat{\ell}$ is now no longer a state variable)

$$
\hat{V}=\psi(1-\psi)^{\frac{1}{\psi}-1} \hat{w}^{1-\frac{1}{\psi}} \hat{Y}+\frac{\beta}{1+g} \hat{V}
$$

Thus,

$$
\hat{V}=\frac{\psi}{1-\beta /(1+g)}(1-\psi)^{\frac{1}{\psi}-1} \hat{w}^{1-\frac{1}{\psi}} \hat{Y}
$$


Note that a constant value of $\hat{\ell}^{\prime}$ implies $\ell_{t}=\ell_{t-1} /(1+g)$. Thus, $\ell_{t-1}-\ell_{t}=g \ell_{t}$ and the firm fires a proportion $g$ of its employees every period.

From the free-entry condition,

$$
\eta^{-1}=\frac{\psi}{1-\beta /(1+g)}(1-\psi)^{\frac{1}{\psi}-1} \hat{w}^{1-\frac{1}{\psi}} \hat{Y}
$$

holds, because $\hat{V}$ here corresponds to $\mathbf{V}_{t} / N_{t}^{\frac{\psi}{1-\psi}-1}$ in (12).

In the case with no tax, the employment $\ell_{t}$ is the same across different goods. From the production function,

$$
Y_{t}=N_{t}^{\frac{1}{1-\psi}} \ell_{t}
$$

and thus

$$
\hat{Y}=\left(\frac{1-\psi}{\hat{w}}\right)^{\frac{1}{\psi}} \hat{Y}
$$

which implies

$$
\hat{w}=1-\psi .
$$

Therefore, the free-entry condition (16) can be rewritten as

$$
\hat{Y}=\frac{1-\beta /(1+g)}{\psi \eta} .
$$

Note this equation represents a positive relationship between $\hat{Y}$ and $g$ because the profit for each intermediate-good producer contracts over time due to increase in $N_{t}$. When $N_{t}$ is large, many intermediate-good firms compete for limited production resources (labor in the current model), which implies the wage increases as $N_{t}$ becomes larger. When $N_{t}$ grows faster, the future profit shrinks faster due to the wage increase. One can interpret this as a form of the creative-destruction effect, because the creation of new varieties forces existing firms to contract. Thus, when $g$ is large, a large $\hat{Y}$ (which supports a larger flow profit) is necessary in order to satisfy the free-entry condition.

Because firms are symmetric, the aggregate labor can be written as

$$
L_{t}=\ell_{t} N_{t} \text {. }
$$

Because $\hat{\ell}=\ell_{t} / N_{t}^{-1}=\ell_{t} N_{t}$, this equation implies $L_{t}=\hat{\ell}$. From (17) together with $\hat{Y}=Y_{t} / N_{t}^{\frac{\psi}{1-\psi}}$, we then have

$$
\hat{Y}=\hat{\ell}=L_{t} .
$$

The growth rate is determined by the $R \& D$ input in (13). To determine the R\&D input, we use the condition $\hat{R}=\hat{Y}-\hat{C}$ and the consumer's static first-order condition

$$
\hat{C}=\frac{\hat{w}}{\xi},
$$

yielding

$$
\hat{R}=\frac{1-\beta /(1+g)}{\psi \eta}-\frac{1-\psi}{\xi} .
$$

Thus, because $g=\eta \hat{R}$ from (13),

$$
g=\frac{1-\beta /(1+g)}{\psi}-\frac{\eta(1-\psi)}{\xi} .
$$




\section{E.5.2 Computation of the model with firing taxes}

At each point in time, the normalized number of firms at age $s, f_{s}$, is

$$
f_{s} \equiv \frac{g N_{t-s-1}}{N_{t}}=\frac{g}{(1+g)^{1+s}} .
$$

The computation proceeds as follows.

1. Guess $g$ and $\hat{w}$. Because $g=\eta \hat{R}=\eta(\hat{Y}-\hat{C})$ and $\hat{C}=\hat{w} / \xi$,

$$
\hat{Y}=\frac{g}{\eta}+\frac{\hat{w}}{\xi} .
$$

2. Solve the Bellman equation (15). Let the policy function be $\hat{\ell}^{\prime}=\mathcal{L}(\hat{\ell})$. Then we can calculate the normalized employment for firms of age $s, \hat{\ell}_{s}$, as

$$
\hat{\ell}_{0}=\mathcal{L}(0)
$$

and

$$
\hat{\ell}_{s}=\mathcal{L}\left(\hat{\ell}_{s-1}\right)
$$

for $s=1,2, \ldots$.

3. Check whether the guess is correct by looking at the two equilibrium conditions. First, from the production function, normalized output has to be

$$
\hat{Y}=\left(\sum_{s=0}^{\infty} \hat{\ell}_{s}^{1-\psi} f_{s}\right)^{\frac{1}{1-\psi}},
$$

where $f_{s}$ is calculated by (19). The free-entry condition is

$$
\hat{V}(0)=\eta^{-1}
$$

because $\hat{V}(0)$ corresponds to $\mathbf{V}_{t} / N_{t}^{\frac{\psi}{1-\psi}-1}$ in the notation of (12). Adjust $g$ and $\hat{w}$ until these two conditions are satisfied.

\section{E.5.3 Calibration}

Similar to the baseline model, we calibrate the frictionless economy to match the U.S growth rate and employment rate. The efficiency of innovation $\eta$ is set so that $g_{Y}=$ 0.0148. The disutility labor $\xi$ is set so that $L_{t}=0.613$. We use the same values of $\beta$ and $\psi$ as for the baseline model: $\beta=0.947$ and $\psi=0.2$.

From

$$
1+g=\left(1+g_{Y}\right)^{\frac{1-\psi}{\psi}}
$$

from 14 , we can obtain $g=0.0605$. Then because

$$
L_{t}=\hat{Y}=\frac{1-\beta /(1+g)}{\psi \eta},
$$

with the target $L_{t}=0.613$, we can obtain $\eta=0.873$. We can solve (18) for $\xi$ and obtain $\xi=1.471$. 


\section{E.5.4 Results}

We consider two experiments: $\tau=0.3$ and $\tau=1.0$. Table 14 summarizes the results. As in our model in the main text, firing taxes have both level effects and growth effects. The growth rate falls, and the quantitative impact is larger than in the main text, largely because innovation occurs here only through entry, which is negatively affected by $\tau$. The incentive to enter is lower because the total benefit of entry is reduced by the tax. In the model in the main text, incumbents' innovation increases and counteracts this effect, whereas here we assume incumbents do not innovate on their own variety.

Table 14: Results: expanding-variety model

\begin{tabular}{|l|r|r|r|}
\hline & $\tau=0.0$ & $\tau=0.3$ & $\tau=1.0$ \\
\hline \hline Growth rate of output $g_{Y}(\%)$ & 1.48 & 1.31 & 1.05 \\
Employment $L$ & 100 & 99.0 & 98.7 \\
Normalized output $\hat{Y}$ & 100 & 98.9 & 98.1 \\
Normalized average productivity $\hat{Y} / L$ & 100 & 99.9 & 99.4 \\
\hline
\end{tabular}

Note: $L, \hat{Y}$, and $\hat{Y} / L$ are set at 100 in the $\tau=0.0$ case.

The level effect on employment and normalized output are also negative. As for the baseline model, this outcome is not trivial. Entrants are smaller than in the frictionless case, but after entering, firms do not fire workers for some time, and eventually they will become larger than in the frictionless case (recall that in the frictionless case, the firm size becomes smaller over time). After a certain point, the firms start firing workers at a constant rate (at the rate $g$, as in the frictionless case). Depending on the size of entrants, the size at which firms start firing workers, and the change in the overall growth rate, the aggregate labor demand can be higher or lower. Labor supply can also change because of the wealth effect.

The average productivity $\hat{Y} / L$ falls because of misallocation, as in the main text. The production function (11) implies the maximum production given $L$ is achieved when $\hat{\ell}$ is constant across firms. Firing taxes generate dispersion in $\hat{\ell}$ (and thus the marginal product of labor) across firms and thus reduces aggregate productivity.

Overall, the expanding-variety model produces similar results to the quality-ladder model in our baseline model. The largest difference is that here we assume the incumbents do not innovate. As a consequence, the effects that are intrinsic to incumbents, such as the tax-escaping effect, are not present in the current model.

\section{F Empirical analysis}

As explained in section III.B, the overall effect on growth is the result of two opposing effects. Firing costs may increase the incumbents' innovation while discouraging the innovation by entrants. The overall effect could be positive or negative depending on which of these two effects dominate. To gain further insight into this question, we conduct in this section an empirical analysis of the effect of firing costs on innovation. Several studies have shown the effects of firing costs on job reallocation (Micco and Pagés, 2007; 
Haltiwanger, Scarpetta and Schweiger, 2014; Davis and Haltiwanger, 2014), but only a few studies have investigated the consequences of firing costs for aggregate productivity. Using differences across the US states in the adoption of more stringent labor laws, Autor, Kerr and Kugler (2007) find evidence suggesting firing costs reduce total factor productivity. More closely related to our objective, Bassanini, Nunziata and Venn (2009) investigate the effects of firing costs on total factor productivity growth. They find that more stringent dismissal regulations tend to reduce total factor productivity growth in industries in which dismissal regulations are more likely to be binding.

In this section, we complement the existing studies by focusing on innovation spending. We analyze two different empirical models. First, we exploit the variation in employment protection regulations across countries and over time to evaluate how industrylevel R\&D spending is correlated with these regulations. Second, we exploit the variation across industries as well and conduct an analysis similar to Bassanini, Nunziata and Venn (2009).

\section{F.1 Data}

R\&D spending (R\&D): We compute R\&D as R\&D business expenditures, divided by the gross output of the industry. We use data on $R \& D$ business expenditures by industry and by country from the OECD ANBERD database (Analytical Business Enterprise Research and Development). The data are available at the two-digit ISIC Rev.3 level and are classified in industries according to the main activity of the enterprise carrying out the R\&D. We remove the financial-intermediation sector from the dataset. The ANBERD dataset includes statistical estimates, which leads to fewer missing values and more extensive time series than the raw data. The ANBERD dataset covers 32 OECD countries and six non-member countries between 1987 and 2011, with gaps and breaks in some of the series. The gross output data, obtained from the OECD STAN database, are also available at the two-digit ISIC Rev.3 level.

Employment protection indicator (EPL): We use two indicators of the strictness of employment protection constructed by the OECD. The indicator EPL1 measures the strictness of dismissal regulation for individual dismissal, and the indicator EPL2 includes measures of the strictness of the regulation on collective dismissal as well. ${ }^{7}$ The indicators are constructed from the reading of statutory laws, collective bargaining agreements, and case law combined with advice from officials from OECD member countries and country experts. The indicators are compiled from scores between 0 and 6 on the notification procedure, the severance pay, and the difficulty of dismissal. The indicator EPL1 is available between 1985 and 2013, and EPL2 is available between 1998 and 2013. The dataset covers 34 OECD countries and 38 non-OECD countries (for most non-OECD countries, the series is not available before 2008). The Employmentprotection indicators are publicly available at http://stats.oecd.org/, and a comprehensive description of the method used to construct the indicator can be found at http://www.oecd.org/els/emp/oecdindicatorsofemploymentprotection.htm.

\footnotetext{
${ }^{7}$ The OECD codes for EPL1 and EPL2 are EPRC_V1 and EPRC_V2.
} 
Layoff rate (layoff): To measure the sensitivity of each industry to firing costs, we use the layoff rate by industry in the US. Dismissal regulation in the US is less strict than in the rest of the countries considered. The US layoff rate can therefore be used as a proxy for the propensity of each industry to lay off workers. Following Bassanini, Nunziata and Venn (2009), we estimate the US layoff rate by industry using data from the 2004 "Displaced workers, Employee, Tenure and Occupational Mobility" supplement of the Current Population Survey (CPS). We measure the layoff rate as the total number of displaced workers in the three years preceding the survey $(2001,2002$ and 2003) divided by total employment in the industry in January 2004. A displaced worker is a worker who has lost his job for one of the following reasons: "plant closing," "insufficient work," "position abolished," "seasonal job ended," or "self-operated business failed." We use the Uniform Extract of CPS made available by the Center for Economic and Policy Research (http://ceprdata.org/cps-uniform-data-extracts/cpsdisplaced-worker-survey/cps-dws-data/). The data are organized according to the 2002 census industry classification. To be consistent with the R\&D data, we convert the layoff data into the two-digit ISIC Rev. 3 classification. The correspondence between the two classifications is reported in Table 15. Though the exact procedure used to estimate the US layoff rate differs from Bassanini, Nunziata and Venn (2009), the two measures are strongly correlated (correlation coefficient of 0.71).

The merged dataset contains data on 27 OECD countries and 19 industries between 1987 and 2009, with breaks and gaps in the series. The 27 countries are: Austria, Belgium, Canada, Czech Republic, Estonia, Finland, Germany, Greece, Hungary, Iceland, Ireland, Israel, Italy, Japan, Korea, Luxembourg, Mexico, Netherlands, New Zealand, Norway, Poland, Portugal, Slovak Republic, Slovenia, Spain, Switzerland, and the US. We excluded the primary sectors, the financial-intermediation industry, as well as public and personal services (education, health, etc). The 19 industries used are listed in Table 15.

\section{F.2 Empirical specifications}

First, we utilize the variation in employment-protection regulations across countries and over time to evaluate how R\&D spending is correlated with these regulations. We estimate the following equation at the industry level:

$$
\log \left(\mathrm{R} \& \mathrm{D}_{j c t}\right)=\beta_{0}+\beta_{1} \mathrm{EPL}_{c t}+\gamma_{j}+\varepsilon_{j c t},
$$

where $\gamma_{j}$ is the industry fixed effect. $R \& \mathrm{D}_{j c t}$ is the R\&D spending of industry $j$ in country $c$ and year $t$, computed as the share of the industry's output, and $\mathrm{EPL}_{c t}$ is the indicator of employment protection. A high value of $\mathrm{EPL}_{c t}$ indicates dismissal regulation is strict and it is thus more costly to fire workers. The parameter of interest is $\beta_{1}$, which indicates how $R \& D$ spending is related to the strictness of employment-protection regulation.

Second, we follow the approach used by Bassanini, Nunziata and Venn (2009). We test whether industries that have a higher propensity to lay off workers have relatively lower R\&D spending in countries where firing costs are high. Cross-industry variation is used to identify the effect of the regulation, with the underlying assumption that 
Table 15: CPS-OECD industry classification correspondence

\begin{tabular}{|c|c|c|c|}
\hline code & CPS label & code & OECD label \\
\hline 4 & Construction & $\mathrm{F}$ & Construction \\
\hline 5 & Nonmetallic mineral product manufacturing & 26 & Non-metallic mineral products \\
\hline 6 & Primary metals and fabricated metal products & $27-28$ & Basic metals and fabricated metal \\
\hline 7 & Machinery manufacturing & 29 & Machinery n.e.c. \\
\hline 8 & Computer and electronic product manufacturing & $30-33$ & Electrical and optical equipment \\
\hline 9 & Electrical equipment, appliance manufacturing & $30-33$ & Electrical and optical equipment \\
\hline 10 & Transportation equipment manufacturing & $34-35$ & Transport equipment \\
\hline 11 & Wood products & 20 & Wood and wood products \\
\hline 12 & Furniture and fixtures manufacturing & $36-37$ & Manufacturing, n.e.c.; recycling \\
\hline 13 & Miscellaneous and not specified manufacturing & $36-37$ & Manufacturing, n.e.c.; recycling \\
\hline 14 & Food manufacturing & $15-16$ & Food and beverages \\
\hline 15 & Beverage and tobacco products & $15-16$ & Food and beverages \\
\hline 16 & Textile, apparel, and leather manufacturing & $17-19$ & Textiles, wearing app. and leather \\
\hline 17 & Paper and printing & $21-22$ & Paper, printing and publ \\
\hline 18 & Petroleum and coal products manufacturing & 23 & Coke, refined petroleum, nuclear fuel \\
\hline 19 & Chemical manufacturing & 24 & Chemicals and chemical products \\
\hline 20 & Plastics and rubber products & 25 & Rubber and plastics \\
\hline 21 & Wholesale trade & $50-52$ & Trade \\
\hline 22 & Retail trade & $50-52$ & Trade \\
\hline 23 & Transportation and warehousing & $60-64$ & Transport, storage and communications \\
\hline 24 & Utilities & $\mathrm{E}$ & Electricity, gas and water supply \\
\hline 25 & Publishing industries (except internet) & $60-64$ & Transport, storage and communications \\
\hline 26 & Motion picture and sound recording industries & $60-64$ & Transport, storage and communications \\
\hline 27 & Broadcasting (except internet) & $60-64$ & Transport, storage and communications \\
\hline 28 & Internet publishing and broadcasting & $60-64$ & Transport, storage and communications \\
\hline 29 & Telecommunications & $60-64$ & Transport, storage and communications \\
\hline 30 & Internet service providers and data processing services & $60-64$ & Transport, storage and communications \\
\hline 31 & Other information services & $60-64$ & Transport, storage and communications \\
\hline 34 & Real estate & $70-74$ & Real estate and business services \\
\hline 35 & Rental and leasing services & $70-74$ & Real estate and business services \\
\hline 36 & Professional and technical services & $70-74$ & Real estate and business services \\
\hline 37 & Management of companies and enterprises & $70-74$ & Real estate and business services \\
\hline 38 & Administrative and support services & $70-74$ & Real estate and business services \\
\hline 45 & Accommodation & $\mathrm{H}$ & Hotels and Restaurants \\
\hline 46 & Food services and drinking places & $\mathrm{H}$ & Hotels and Restaurants \\
\hline
\end{tabular}

Notes: The CPS classification is the 2002 Census Industry Classification and the OECD classification is ISIC Rev.3. 
Table 16: Regression results: $\log (\mathrm{R} \& \mathrm{D}$ ratio $)$

\begin{tabular}{l|ccc|ccc}
\hline \hline & \multicolumn{3}{|c|}{ Individual dismissal } & \multicolumn{3}{c}{ Individual and collective dismissal } \\
& {$[1]$} & {$[2]$} & {$[3]$} & {$[1]$} & {$[2]$} & {$[3]$} \\
\hline $\mathrm{EPL}_{c t}$ & $-0.461^{* * *}$ & $-0.441^{* * *}$ & $-0.461^{* * *}$ & $-0.656^{* * *}$ & $-0.754^{* * *}$ & $-0.656^{* * *}$ \\
& $(0.0148)$ & $(0.0197)$ & $(0.0148)$ & $(0.0255)$ & $(0.0304)$ & $(0.0255)$ \\
\hline$N$ & 5755 & 3055 & 5755 & 3770 & 2233 & 3770 \\
$R^{2}$ & 0.552 & 0.531 & 0.552 & 0.577 & 0.577 & 0.577 \\
\hline \hline
\end{tabular}

Standard errors in parentheses

${ }^{*} p<0.05,{ }^{* *} p<0.01,{ }^{* * *} p<0.001$

Notes: The columns refer to different samples: [1] non-balanced panel [2] balanced panel [3] year $=2005$. The balanced panel contains data on 18 countries and 19 industries from 1995 to 2005. All regressions include industry fixed effects. Robust standard errors in parentheses. * $p<0.05 ;{ }^{* *} p<0.01{ }^{* * *} p<0.001$.

industries with a higher layoff propensity are more sensitive to firing costs. This strategy greatly reduces the concerns about omitted variable bias because it allows us to control for both country and industry fixed effects. Hence, our results cannot be driven by other cross-country differences in regulations or policies as long as they do not affect industries with different layoff propensities differently. We estimate the following equation:

$$
\log \left(\mathrm{R} \& \mathrm{D}_{j c t}\right)=\beta_{0}+\beta_{1} \mathrm{EPL}_{c t} \times \log \left(\text { layoff }_{j}\right)+\gamma_{j}+\lambda_{c t}+\varepsilon_{j c t},
$$

where $\lambda_{c t}$ is the country-time fixed effect. The indicator of the industry's propensity to lay off workers layoff ${ }_{j}$ corresponds to the industry's layoff rate in the absence of any dismissal regulation. The parameter of interest here is that of the interaction between the level of employment protection and the industry's propensity to lay off workers $\beta_{1}$. When $\beta_{1}<0$, countries with stricter dismissal regulation have relatively lower $\mathrm{R} \& \mathrm{D}$ spending in industries with a higher propensity to lay off workers. Conversely, $\beta_{1}>0$ would indicate countries with stricter dismissal regulation have relatively higher $R \& D$ spending in industries with a higher propensity to lay off workers.

\section{F.3 Empirical Results}

The results of the OLS estimation of equation (20) are displayed in Table 16 for the two measures of employment protection, EPL1 and EPL2. The first column reports the results for the full sample. Because the data have missing observations for some industries and some countries, we also run the regression on the balanced panel (column [2]) and for a given year (column [3]) to ensure the missing observations do not bias the results. All six regressions indicate $\mathrm{R} \& \mathrm{D}$ spending is negatively correlated with employment-protection regulation.

The OLS estimation results of equation (21) are in Table 17. The interaction terms in all specifications have insignificant coefficients. Employment-protection legislation does not have a systematically larger effect in industries with a higher layoff rate. From 
Table 17: Regression results: $\log (\mathrm{R} \& \mathrm{D}$ ratio), with $\log ($ layoff rate) as a proxy

\begin{tabular}{l|ccc|ccc}
\hline \hline \multirow{2}{*}{} & \multicolumn{3}{|c|}{ Individual dismissal } & \multicolumn{3}{c}{ Individual and collective dismissal } \\
& {$[1]$} & {$[2]$} & {$[3]$} & {$[1]$} & {$[2]$} & {$[3]$} \\
& {$[1]$} & EPL2 & 0.388 \\
$\mathrm{EPL}_{c t} \times \ln \left(\right.$ layoff $\left._{j}\right)$ & 0.0303 & 0.132 & 0.249 & 0.139 & 0.158 & $(0.334)$ \\
& $(0.0578)$ & $(0.0861)$ & $(0.251)$ & $(0.102)$ & $(0.123)$ & 343 \\
$N$ & 5755 & 3055 & 343 & 3770 & 2233 & 0.777 \\
$R^{2}$ & 0.772 & 0.775 & 0.776 & 0.781 & 0.775 & \\
\hline \hline
\end{tabular}

Standard errors in parentheses

${ }^{*} p<0.05,{ }^{* *} p<0.01,{ }^{* * *} p<0.001$

Notes: The layoff rate is measured from the CPS displacement data. The columns refer to different samples: [1] non-balanced panel [2] balanced panel [3] year $=2005$. The balanced panel contains data on 18 countries and 19 industries from 1995 to 2005. The non-balanced and balanced panel regressions include industry and country-time fixed effects. The 2005 regression incudes industry and country fixed effects. Robust standard errors in parentheses. ${ }^{*} p<0.05 ;{ }^{* *} p<0.01 ;{ }^{* * *} p<0.001$.

Table 18: Regression results: $\log (\mathrm{R} \& \mathrm{D}$ ratio $)$, with $\log ($ job destruction rate) as a proxy

\begin{tabular}{l|ccc|ccc}
\hline \hline \multirow{2}{*}{} & \multicolumn{3}{|c|}{ Individual dismissal } & \multicolumn{3}{c}{ Individual and collective dismissal } \\
& {$[1]$} & {$[2]$} & {$[3]$} & {$[1]$} & {$[2]$} & {$[3]$} \\
\hline $\mathrm{EPL}_{c t} \times \ln$ jdrate $_{j}$ & -0.0589 & -0.00950 & 0.424 & 0.0469 & $-0.246^{*}$ & 0.356 \\
& $(0.0507)$ & $(0.0628)$ & $(0.242)$ & $(0.0797)$ & $(0.101)$ & $(0.329)$ \\
\hline$N$ & 5392 & 2844 & 320 & 3516 & 2080 & 320 \\
$R^{2}$ & 0.766 & 0.767 & 0.735 & 0.756 & 0.760 & 0.732 \\
\hline \hline
\end{tabular}

Standard errors in parentheses

${ }^{*} p<0.05,{ }^{* *} p<0.01,{ }^{* * *} p<0.001$

Notes: The job destruction rate is measured using data made available by John Haltiwanger. The columns refer to different samples: [1] non-balanced panel [2] balanced panel [3] year $=2005$. The balanced panel contains data on 18 countries and 15 industries from 1995 to 2005. The non-balanced and balanced panel regressions include industry and country-time fixed effects. The 2005 regression incudes industry and country fixed effects. Robust standard errors in parentheses. ${ }^{*} p<0.05 ;^{* *} p<0.01 ;{ }^{* * *} p<0.001$. 
the viewpoint of our theoretical model, the positive and negative effects of employment protection on R\&D may offset each other to produce mixed results.

As a robustness check, we use the job-destruction rate instead of the layoff rate as a proxy for the industries' sensitivity to firing costs (both computed on US data). The data, made available online by John Haltiwanger (Bartelsman, Haltiwanger and Scarpetta, 2009), span the periods 1989-1991 and 1994-1996. The industry classification is derived from the STAN classification, but at a higher aggregation level than the R\&D data. We re-aggregate the $R \& D$ data at the level at which the job-destruction rate is available. The merged dataset has 17 industries (vs. 19 with our original dataset). The results, reported in Table 18, show the coefficient of the interaction term is insignificant for most regressions, similar to what we obtained when using the layoff rate. The one significant coefficient is negative, consistently with the result of the entrant-driven calibration.

All in all, the empirical results only suggest a negative effect of firing costs on innovation spending. We find that countries with stricter dismissal regulations tend to invest less in R\&D, but this effect does not hold once we control for country fixed effects and use the cross-industry variation to identify the effect of the dismissal regulation.

\section{References}

Autor, David H., William R. Kerr, and Adriana D. Kugler. 2007. "Do Employment Protections Reduce Productivity? Evidence from U.S. States." Economic Journal, 117: F189-F217.

Axtell, Robert L. 2001. "Zipf Distribution of U.S. Firm Sizes." Science, 293: 18181820 .

Bartelsman, Eric, John Haltiwanger, and Stefano Scarpetta. 2009. "Measuring and Analyzing Cross-country Differences in Firm Dynamics." In Producer Dynamics: New Evidence from Micro Data. NBER Chapters, 15-76. National Bureau of Economic Research, Inc.

Bassanini, Andrea, Luca Nunziata, and Danielle Venn. 2009. "Job protection legislation and productivity growth in OECD countries." Economic Policy, 24: 349402.

Bena, Jan, Lorenzo Garlappi, and Patrick Grüning. 2015. "Heterogeneous Innovation, Firm Creation and Destruction, and Asset Prices." Review of Asset Pricing Studies, 6: 46-87.

Davis, Steven J., and John Haltiwanger. 2014. "Labor Market Fluidity and Economic Performance." NBER Working Papers 20479.

Haltiwanger, John, Stefano Scarpetta, and Helena Schweiger. 2014. "Cross country differences in job reallocation: The role of industry, firm size and regulations." Labour Economics, 26: 11-25.

Lucas, Robert E. 1987. Models of Business Cycles. New York: Basil Blackwell. 
Micco, Alejandro, and Carmen Pagés. 2007. "The Economic Effects of Employment Protection: Evidence from International Industry-level Data." mimeo.

Ohanian, Lee, Andrea Raffo, and Richard Rogerson. 2008. "Long-Term Changes in Labor Supply and Taxes: Evidence from OECD Countries, 1956-2004." Journal of Monetary Economics, 55: 1353-1362.

Rogerson, Richard, and Johanna Wallenius. 2009. "Micro and Macro Elasticities in a Life Cycle Model with Taxes." Journal of Economic Theory, 133: 2277-2202.

U.S. Census Bureau. 2011. "Synthetic LBD Beta Version 2.0." U.S. Census Bureau and Cornell University, Synthetic Data Server [distributor] [Computer file], Washington,DC and Ithaca, NY, USA. 